\title{
Harnessing Direct(Hetero)Arylation in Pursuit of a Saddle-Shaped Perylene Diimide Tetramer
}

(Supporting Information)

\author{
Josh D. B. Koenig, Audrey Laventure, and Gregory C. Welch ${ }^{\text {a* }}$ \\ ${ }^{a}$ Department of Chemistry, University of Calgary, 2500 University Drive N.W., Calgary, \\ Alberta, Canada T2N 1N4 \\ * Corresponding Author \\ Email: gregory.welch@ucalgary.ca;
}

Phone number: 1-403-210-7603

Keywords: saddle-shaped; perylene diimide; tetramer; direct(hetetro)arylation; DHA

\section{TABLE OF CONTENTS}

1. Methods and Materials

S2-S3

2. Synthetic/Experimental Procedures

S4-S5

3. ${ }^{1} \mathrm{H} \&{ }^{13} \mathrm{C}$ NMR Spectroscopy

S6-S9

4. HR MALDI-TOF

S10-S11

5. Elemental Analysis \& Thermal Properties

S12

6. UV-Visible Spectroscopy \& X-Ray Diffraction

S13-S15

7. Voltammetry

S16

8. Density Functional Theory

S17

9. OPV Device Optimization

S18-S22

10. References

S23 


\section{Methods and Materials}

Materials: Polymer PTB7-Th was purchased from 1-Material. SiliaCat@ DPP-Pd was purchased from SiliCycle. All other reactants, reagents, and catalysts were purchased from Sigma-Aldrich or VWR and used without further purification.

CHN Elemental Analysis: Elemental analyses were performed by Johnson Li in the Chemical Instrumentation Facility at the University of Calgary. A Perkin Elmer 2400 Series II CHN Elemental Analyzer was used to obtain CHN data, using $1.5 \mathrm{mg}$ of sample (with particle sizes ranging between 0.2 and $0.5 \mathrm{~mm}$ in diameter).

Nuclear Magnetic Resonance (NMR): ${ }^{1} \mathrm{H}$ and ${ }^{13} \mathrm{C}$ NMR spectroscopy experiments were recorded using either a Bruker Avance III 500 or $600 \mathrm{MHz}$ spectrometer. All experiments were performed in either chloroform-d $\left(\mathrm{CDCl}_{3}\right)$ or tetrachloroethane- $\mathrm{d}_{2}$. Chemical shifts (referenced to residual solvent) were reported in parts per million ( $\mathrm{ppm})$. Multiplicities were reported as follows: singlet (s), doublets (d), triplets (t), quartet (q), pentet (p), hextet (h), doublet of doublets (dd), doublet of triplets $(\mathrm{dt})$, doublet of quartets $(\mathrm{dq})$, triplet of doublets (td), triplet of triplets (tt), and multiplets (m).

High-resolution MALDI-TOF (HR MALDI-TOF): High-resolution MALDI-TOF mass spectrometry measurements were performed courtesy of Jian Jun (Johnson) Li in the Chemical Instrumentation Facility at the University of Calgary. The sample solution $(\sim 1 \mu \mathrm{g} / \mathrm{ml}$ in dichloromethane) was mixed with matrix trans2-[3-(4-tert-Butylphenyl)-2-methyl-2propenylidene]malononitrile (DCTB) solution $(\sim 5 \mathrm{mg} / \mathrm{ml}$ in methanol). All spectra were acquired using a Bruker Autoflex III Smartbeam MALDI-TOF, set to the positive reflective mode (Na:YAG $355 \mathrm{~nm}$ laser settings: laser offset $=62-69$; laser frequency $=200 \mathrm{~Hz}$; and number of shots $=300$ ). The target used was Bruker MTP 384 ground steel plate target.

UV-Visible Spectroscopy (UV-Vis): All absorption measurements were recorded using Agilent Technologies Cary $60 \mathrm{UV}-\mathrm{V}$ is spectrometer at room temperature. All solution UV-Vis spectra were measured with $2 \mathrm{~mm}$ quartz cuvettes, using $\mathrm{CH}_{2} \mathrm{Cl}_{2}$ as solvent. Stock solutions $(1.0 \mathrm{mg} / \mathrm{mL})$ of each compound were prepared, serially diluted to concentrations between $10^{-5}-10^{-6} \mathrm{M}$, and then used to construct calibration curves for determining molar absorptivity. Variable-temperature measurements were performed using an Agilent Technologies Cary single cell Peltier accessory. Neat films were prepared by spin-coating from a $1 \% \mathrm{wt} / \mathrm{v}$ solution onto clean Corning glass micro slides. Prior to use, glass slides were cleaned with soap and water, acetone and isopropanol, and followed by UV/ozone treatment using a Novascan UV/ozone cleaning system.

Photoluminescence (PL): All emission measurements were recorded using an Agilent Technologies Cary Eclipse fluorescence spectrophotometer at room temperature.

Atomic Force Microscopy (AFM): AFM measurements were performed by using a TT2-AFM (AFM Workshop) in tapping mode and WSxM software with an 0.01-0.025 Ohm/cm Sb (n) doped $\mathrm{Si}$ probe with a reflective back side aluminum coating. Samples for AFM measurement were the same ones that were used to collect the respective the device parameters. 
X-Ray Diffraction (XRD):_All X-ray diffraction experiments were performed with a PROTO AXRD Benchtop Powder Diffractometer using $\theta-2 \theta$ scans and $\mathrm{Cu} K-\alpha$ radiation

Cyclic Voltammetry (CV): Electrochemical measurements were performed using a $\mathrm{CH}$ Instruments Inc. Model 1200B Series Handheld Potentiostat. A standard 3-electrode setup was utilized, consisting of a freshly polished glassy carbon disk working electrode (WE), Pt-wire counter electrode (CE), and Ag-wire pseudo-reference electrode (RE). All measurements were referenced to ferrocene $\left(\mathrm{Fc}^{+/ 0}\right)$ as internal standard. All cyclic voltammetry experiments were performed at a scan rate of $100 \mathrm{mV} / \mathrm{s}$. Sample solutions, with $1 \mathrm{mM}$ compound and $0.1 \mathrm{M}$ tetrabutylammonium hexafluorophosphate $\left(\mathrm{TBAPF}_{6}\right)$ supporting electrolyte, were prepared in anhydrous $\mathrm{CH}_{2} \mathrm{Cl}_{2}$. All electrochemical solutions were sparged with dry gas (either $\mathrm{N}_{2}$ or argon) for 5 minutes to deoxygenate the system prior to measurements. The ionization potentials (IP) and electron affinities (EA) were estimated by correlating the $1^{\text {st }}$ oxidation and $1^{\text {st }}$ reduction potentials $\left(\mathrm{E}_{0 x} 1_{2} \mathrm{Fc}^{0 /+}\right.$, Ered $1 / 2 \mathrm{Fc}^{0 /+}$ ) to the normal hydrogen electrode (NHE), assuming the IP of $\mathrm{Fc}^{0 /+}$ to be $4.80 \mathrm{eV}$, respectively. ${ }^{[1]}$

Power Conversion Efficiency (PCE) and External Quantum Efficiency (EQE): The current density-voltage (J-V) curves were measured by a Keithley 2420 source measure unit. The photocurrent was measured under AM 1.5 illumination at $100 \mathrm{~mW} / \mathrm{cm}^{2}$ under a Solar Simulator (Newport 92251A-1000). The standard silicon solar cell (Newport 91150V) was used to calibrate light intensity. EQE was measured in a QEX7 Solar Cell Spectral Response/QE/IPCE Measurement System (PV Measurement, Model QEX7, USA) with an optical lens to focus the light into an area about $0.04 \mathrm{~cm}^{2}$, smaller than the dot cell. The silicon photodiode was used to calibrate the EQE measurement system in the wavelength range from 300 to $1100 \mathrm{~nm}$.

Organic Photovoltaic (OPV) Devices: Devices were fabricated using ITO-coated glass substrates cleaned by sequentially ultra-sonicating with detergent and de-ionized water, acetone, and isopropanol followed by exposure to UV/ozone for $30 \mathrm{~min}$. $\mathrm{ZnO}$ was subsequently deposited as a sol-gel precursor solution in a $\mathrm{N}_{2}$ purge box following the method of Sun et al. ${ }^{[2]}$ The room temperature solution was spin-coated at a speed of $4500 \mathrm{rpm}$ and then annealed at $200{ }^{\circ} \mathrm{C}$ for at least $15 \mathrm{~min}$. Active layer solutions of PTB7-Th and $\mathrm{Th}_{4} \mathrm{PDI}_{4}$ were prepared in air with a total concentration of $10-20 \mathrm{mg} / \mathrm{mL}$ in $o$-dichlorobenzene (o-DCB) with $0-5 \% \quad(\mathrm{v} / \mathrm{v}) \quad 1-$ chloronaphthalene $(\mathrm{CN})$ additive, unless otherwise noted. Solutions were stirred for $4 \mathrm{~h}$ at room temperature. Active layer materials were combined in a 1:1 donor/acceptor weight ratio, unless otherwise noted. The active layer solution was coated at room temperature at a speed of $1000 \mathrm{rpm}$ for $60 \mathrm{~s}$. The substrates with the cast active layers were kept in an $\mathrm{N}_{2}$ atmosphere glovebox overnight before evaporating $\mathrm{MoO}_{3}$ and $\mathrm{Ag}$. The $10 \mathrm{~nm}$ of $\mathrm{MoO}_{3}$ followed by $100 \mathrm{~nm}$ of Ag were thermally deposited under vacuum $\left(10^{-5}\right.$ Torr $)$. The active areas of the devices were $0.14 \mathrm{~cm}^{2}$.

Computational Details: Gas-phase B3LYP/6-31G(d,p) ground-state equilibrium geometry optimizations were considered within Gaussian 09. ${ }^{[3]}$ To reduce the computational cost, all alkylsubstituents were truncated to methyl groups. Molecular dihedral angles were systematically altered to ensure that the optimized geometric structure possessed no imaginary frequencies (at the same level of theory). TD-SCF calculations were also performed from this optimized geometry. Single point calculations were performed on this structure to generate molecular orbitals and electrostatic potential maps. 


\section{Synthetic/Experimental Procedures}

\section{2,5,8,11-tetrakis(trimethylsilyl)cyclooctatetrathiophene ( $\left.\mathrm{Th}_{4} \mathbf{T M S}_{4}\right)$}

$\mathrm{TMS}_{4} \mathrm{Th}_{4}$ was synthesized by iteratively combining two known literature preparations ${ }^{[4,5]}$.

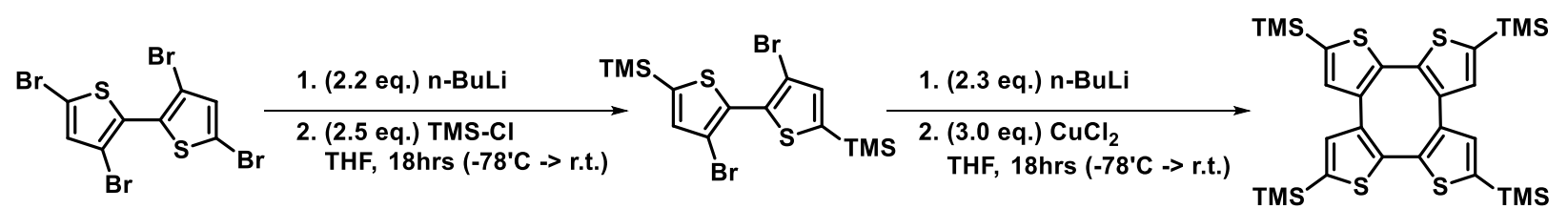

Into a $250 \mathrm{~mL}$ Schlenk flask, add 3,3',5,5'-tetrabromobithiophene (2.0g, $4.16 \mathrm{mmol}, 1$ eq.) and seal the flask with a septum. Complete three vacuum purge and nitrogen backfill cycles on the Schlenk line. Once filled with $\mathrm{N}_{2}$, cannula transfer over dried THF $(80 \mathrm{~mL})$. Stir to dissolve the $3,3^{\prime}, 5,5^{\prime}$-tetrabromobithiophene and then cool reaction mixture to $-78^{\circ} \mathrm{C}$. Dropwise add $n$ butyllithium ( $3.7 \mathrm{~mL}, 9.2 \mathrm{mmol}, 2.2$ eq.) and leave the reaction to stir for 1 hour. Then trimethylsilylchloride ( $1.3 \mathrm{~mL}, 10.4 \mathrm{mmol}, 2.5 \mathrm{eq}$.) was added dropwise to the cooled solution and left to stir overnight. The stirring reaction mixture (containing in-situ generated intermediate) was cooled to $-78^{\circ} \mathrm{C}$. $n$-butyllithium $(4.0 \mathrm{~mL}, 9.8 \mathrm{mmol}, 2.3 \mathrm{eq}$.) was dropwise added and left to stir for 1 hour. Under a stream of $\mathrm{N}_{2}$, the septum was opened and $\mathrm{CuCl}_{2}(1.70 \mathrm{~g}, 12.6 \mathrm{mmol}, 3$ eq.) was added to the reaction. The system was re-sealed and lef to stir overnight. The reaction was quenched with water $(75 \mathrm{~mL})$, and then extracted using diethyl ether (3 x $50 \mathrm{~mL})$. Diethyl ether extracts were dried over $\mathrm{Na}_{2} \mathrm{SO}_{4}$. The drying agent was filtered off and the solvent was concentrated under reduced pressure. The resulting crude oil was purified by silica gel column chromatography (eluted with hexanes) to afford pale yellow solid (452 mg, $0.73 \mathrm{mmol}, \underline{35 \%}$ ). The isolated compound matched previously reported spectroscopic properties ${ }^{[5]}$.

\section{Cyclooctatetrathiophene ( $\left.\mathbf{T h}_{4}\right)$}

$\mathrm{Th}_{4}$ was synthesized using slightly modified literature procedure ${ }^{[6]}$.

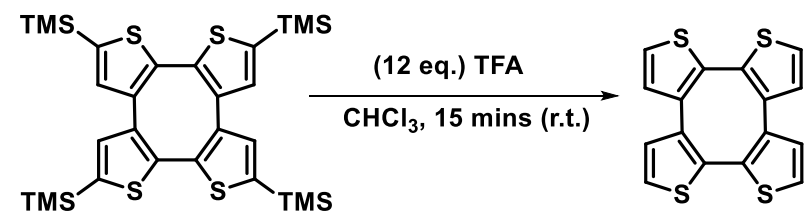

Into a $100 \mathrm{~mL}$ rbf, $\mathrm{Th}_{4} \mathrm{TMS}_{4}\left(300 \mathrm{mg}, 0.48 \mathrm{mmol}, 1\right.$ eq.) was dissolved in minimal $\mathrm{CHCl}_{3}$. Trifluoroacetic acid ( $1 \mathrm{~mL}, 5.9 \mathrm{mmol}, 12 \mathrm{eq}$.) was added dropwise to the rapidly stirring solution. After 15 minutes, the reaction was quenched by adding $\mathrm{H}_{2} \mathrm{O}(40 \mathrm{~mL})$. The aqueous mixture was extracted with $\mathrm{CHCl}_{3}(3 \times 30 \mathrm{~mL})$, and then those extracts were washed with $\mathrm{H}_{2} \mathrm{O}(2 \times 50 \mathrm{~mL})$. After drying organic extracts over $\mathrm{Na}_{2} \mathrm{SO}_{4}$, the extracts were filtered through Celite and solvent was removed under reduced pressure. Precipitating from $\mathrm{MeOH}$, the resulting off-white solid was collected by vacuum filtration $(128 \mathrm{mg}, 0.47 \mathrm{mmol}, \underline{98 \%})$. The isolated compound also matched previously reported spectroscopic properties ${ }^{[6]}$. 
${ }^{1} \mathrm{H} \mathrm{NMR}\left(500 \mathrm{MHz}, \mathrm{CDCl}_{3}\right) \delta 7.38(\mathrm{~d}, J=5.2 \mathrm{~Hz}, 4 \mathrm{H}), 6.97(\mathrm{~d}, J=5.2 \mathrm{~Hz}, 4 \mathrm{H})$.

${ }^{13} \mathrm{C} \mathrm{NMR}\left(126 \mathrm{MHz}, \mathrm{CDCl}_{3}\right) \delta 135.95,131.78,129.33,126.50$.

$\underline{\text { HRMS }}\left([\mathrm{M}]^{+}\right)$calculated for $\mathrm{M}=\mathrm{C}_{16} \mathrm{H}_{8} \mathrm{~S}_{4}: 327.9503$; detected $[\mathrm{M}]^{+}: 327.9518$

\section{Tetrakis(N-hexyl-perylenediimide)cyclooctatetrathiophene $\left(\mathrm{Th}_{4} \mathrm{PDI}_{4}\right)$}

Starting material, hexyl-N-perylenediimide bromide (Hex-N-PDI-Br), was synthesized using previously reported literature procedure ${ }^{[7]}$.
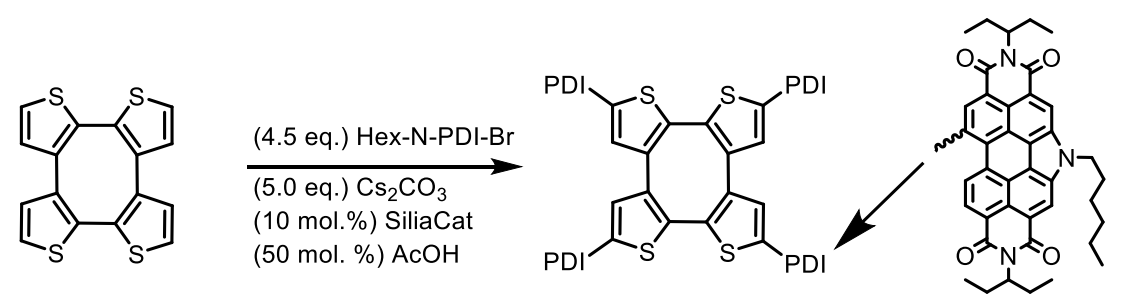

$\mathrm{Th}_{4}$ (32.8 mg, $0.10 \mathrm{mmol}, 1$ eq.), Hex-N-PDI-Br (320 mg, $0.45 \mathrm{mmol}, 4.5$ eq.), $\mathrm{Cs}_{2} \mathrm{CO}_{3}$ (163 mg, $0.50 \mathrm{mmol}, 5$ eq.), and SiliaCat-Pd-DPP $(0.1$ eq., $40 \mathrm{mg}, 0.01 \mathrm{mmol})$ were all added to a $10 \mathrm{~mL}$ $\mu$ wave vial. The vial was sealed and purge with $\mathrm{N}_{2}$ for 30 minutes. Dried dimethylacetamide $(6 \mathrm{~mL})$ was cannula transferred into the vessel and the reaction mixture was sparged for an additional 15 minutes. Acetic acid ( $3 \mathrm{mg}, 0.05 \mathrm{mmol}, 0.5 \mathrm{eq}$.) was injected into the $\mu$ wave vial and then it was placed in a $120^{\circ} \mathrm{C}$ bead bath for 24 hours. The reaction mixture was poured into methanol $(150 \mathrm{~mL})$, stirred for 1 hour, and then vacuum filtered. This crude solid was dissolved in $\mathrm{CHCl}_{3}$, adhered to silica, and further purified by silica gel column chromatography (eluting with $\mathrm{CHCl}_{3}$ ). The resulting crimson red solid was precipitated into methanol and collected by vacuum filtration. (187 mg, $0.07 \mathrm{mmol}, \underline{70 \%})$.

$\underline{{ }^{1} \mathrm{H} \mathrm{NMR}}\left(600 \mathrm{MHz}\right.$, tetrachloroethane- $\left.\mathrm{d}_{2}, 120^{\circ} \mathrm{C}\right) \delta 8.50\left(\mathrm{~s}, 1 \mathrm{H}, \mathrm{H}_{\mathrm{abc}}\right), 8.46\left(\mathrm{~s}, 1 \mathrm{H}, \mathrm{H}_{\mathrm{abc}}\right), 8.41(\mathrm{~s}$, $\left.1 \mathrm{H}, \mathrm{H}_{\mathrm{abc}}\right), 8.31\left(\mathrm{~d}, J=8.3 \mathrm{~Hz}, 1 \mathrm{H}, \mathrm{H}_{\mathrm{d}}\right), 8.00\left(\mathrm{~d}, J=8.3 \mathrm{~Hz}, 1 \mathrm{H}, \mathrm{H}_{\mathrm{e}}\right), 7.10\left(\mathrm{~s}, 1 \mathrm{H}, \mathrm{H}_{\mathrm{f}}\right), 4.64-4.57$ $\left(\mathrm{m}, 1 \mathrm{H}, \mathrm{H}_{\mathrm{g}}\right), 4.38\left(\mathrm{~m}, 1 \mathrm{H}, \mathrm{H}_{\mathrm{h}}\right), 4.32\left(\mathrm{t}, J=7.3 \mathrm{~Hz}, 2 \mathrm{H}, \mathrm{H}_{\mathrm{i}}\right), 1.76\left(\mathrm{dq}, J=15.8,7.9 \mathrm{~Hz}, 2 \mathrm{H}, \mathrm{H}_{\mathrm{j}}\right), 1.69$ $\left(\mathrm{p}, J=7.4 \mathrm{~Hz}, 2 \mathrm{H}, \mathrm{H}_{\mathrm{k}}\right), 1.53\left(\mathrm{tt}, J=16.1,8.1 \mathrm{~Hz}, 2 \mathrm{H}, \mathrm{H}_{\mathrm{l}}\right), 1.45\left(\mathrm{dt}, J=14.1,7.0 \mathrm{~Hz}, 2 \mathrm{H}, \mathrm{H}_{\mathrm{m}}\right), 1.28$ $\left(\mathrm{dq}, J=14.3,7.4 \mathrm{~Hz}, 2 \mathrm{H}, \mathrm{H}_{\mathrm{n}}\right), 0.97\left(\mathrm{p}, J=7.3 \mathrm{~Hz}, 2 \mathrm{H}, \mathrm{H}_{\mathrm{o}}\right), 0.86\left(\mathrm{dt}, J=15.6,6.9 \mathrm{~Hz}, 2 \mathrm{H}, \mathrm{H}_{\mathrm{p}}\right)$, $0.78\left(\mathrm{dt}, J=14.6,7.2 \mathrm{~Hz}, 2 \mathrm{H}, \mathrm{H}_{\mathrm{q}}\right), 0.43\left(\mathrm{t}, J=7.4 \mathrm{~Hz}, 6 \mathrm{H}, \mathrm{H}_{\mathrm{r}}\right), 0.31\left(\mathrm{t}, J=7.3 \mathrm{~Hz}, 3 \mathrm{H}, \mathrm{H}_{\mathrm{s}}\right), 0.24$ $\left(\mathrm{dt}, J=7.4,3.5 \mathrm{~Hz}, 6 \mathrm{H}, \mathrm{H}_{\mathrm{t}}\right)$.

${ }^{13} \mathrm{C}$ NMR $\left(151 \mathrm{MHz}\right.$, tetrachloroethane- $\left.\mathrm{d}_{2}, 120{ }^{\circ} \mathrm{C}\right) \delta 165.17,165.09,163.67,163.57,144.89$, 137.50, 134.81, 134.68, 134.04, 132.64, 132.55, 131.76, 131.15, 129.51, 127.41, 127.02, 124.47, $124.41,122.91,122.78,122.68,122.14,121.82,121.28,119.61,119.51,118.13,117.73,57.70$, $57.33,46.41,30.74,30.67,26.27,24.88,24.69,21.70,12.98,10.73,10.60$.

$\underline{\text { HRMS }}\left([\mathrm{M}+\mathrm{Na}]^{+}\right)$calculated for $\mathrm{M}=\mathrm{C}_{176} \mathrm{H}_{164} \mathrm{~N}_{12} \mathrm{O}_{16} \mathrm{~S}_{4}: 2852.1163$; detected $[\mathrm{M}+\mathrm{Na}]^{+}: 2852.1254$

UV-Vis $\lambda\left(\mathrm{M}^{-1} \mathrm{~cm}^{-1}\right) 298 \mathrm{~nm}(140000), 497 \mathrm{~nm}$ (180000), $534 \mathrm{~nm}$ (210000)

CHN theoretical (\%) C: 74.66, H: 5.84, N: 5.94; found (\%) C: 74.34; H: 5.68; N: 5.80 


\section{3. ${ }^{1} H \&{ }^{13}$ C NMR Spectroscopy}

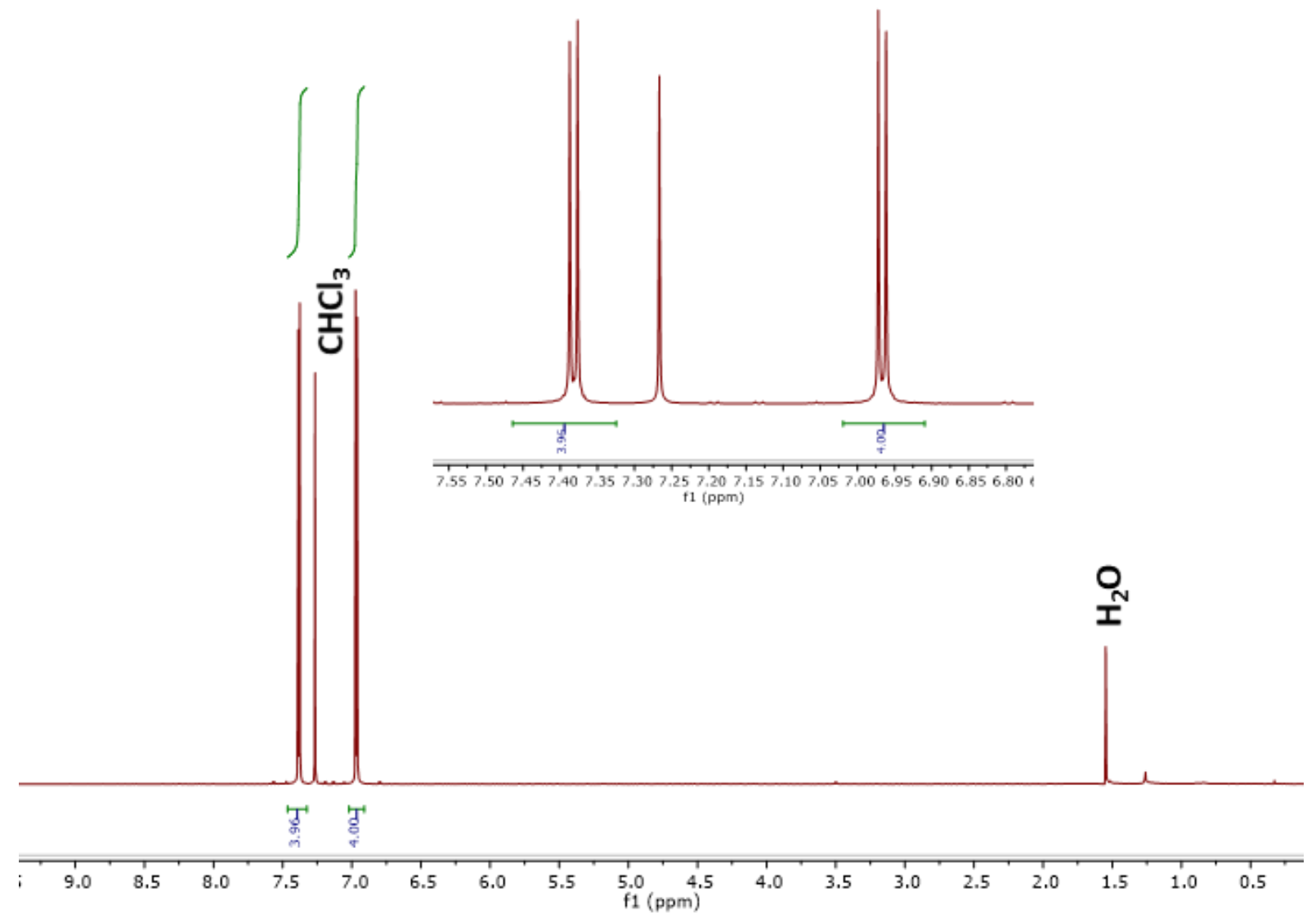

Figure S1. ${ }^{1} \mathrm{H}$ NMR spectrum of $\mathrm{Th}_{4}\left(500 \mathrm{MHz}, \mathrm{CDCl}_{3}\right)$.

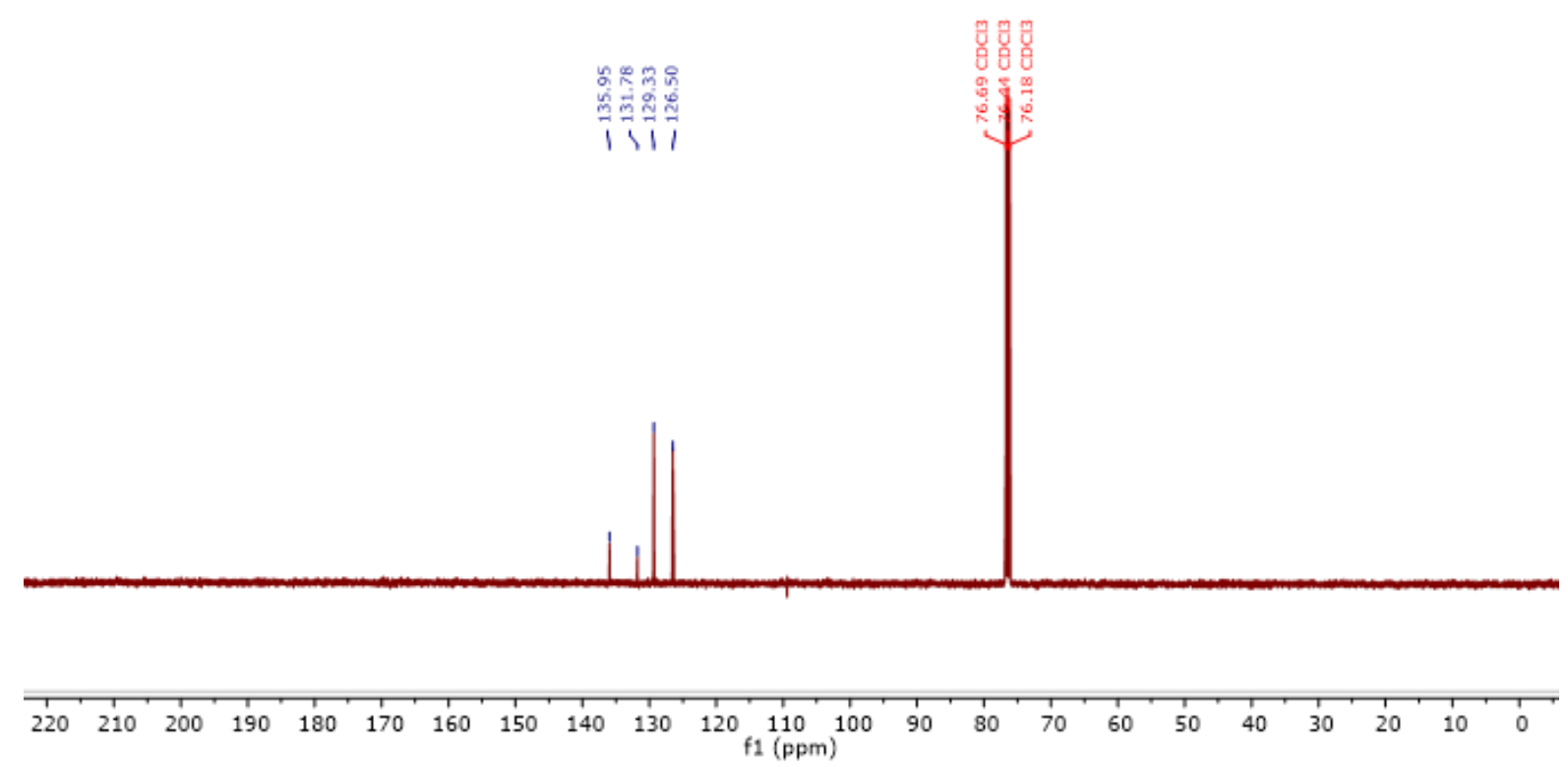

Figure S2. ${ }^{13} \mathrm{C}$ NMR spectrum of $\mathrm{Th}_{4}\left(126 \mathrm{MHz}, \mathrm{CDCl}_{3}\right)$. 


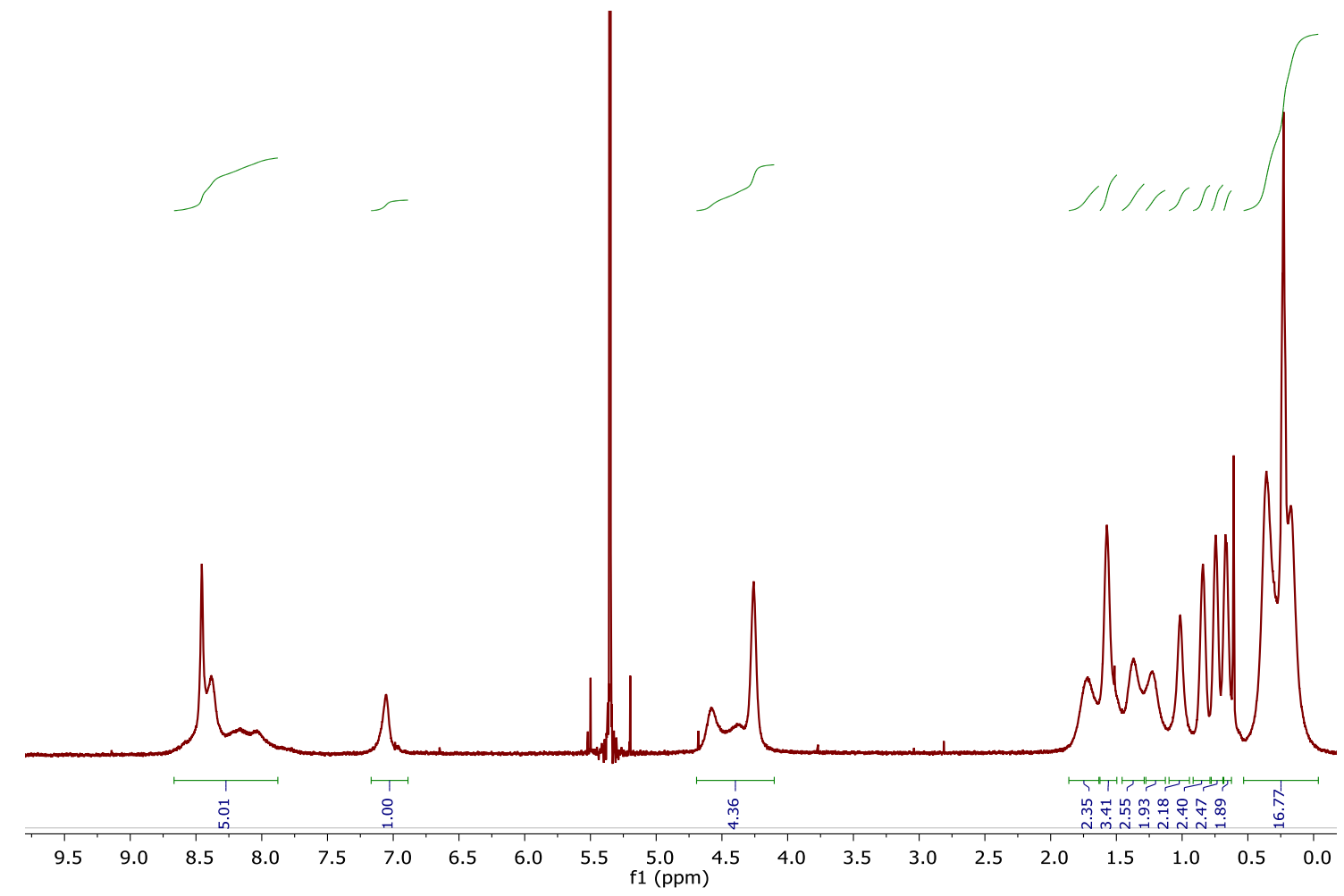

Figure S3. ${ }^{1} \mathrm{H}$ NMR spectrum of $\mathrm{Th}_{4} \mathrm{PDI}_{4}\left(600 \mathrm{MHz}\right.$, tetrachloroethane- $\left.\mathrm{d}_{2}, 2{ }^{\circ} \mathrm{C}\right)$.

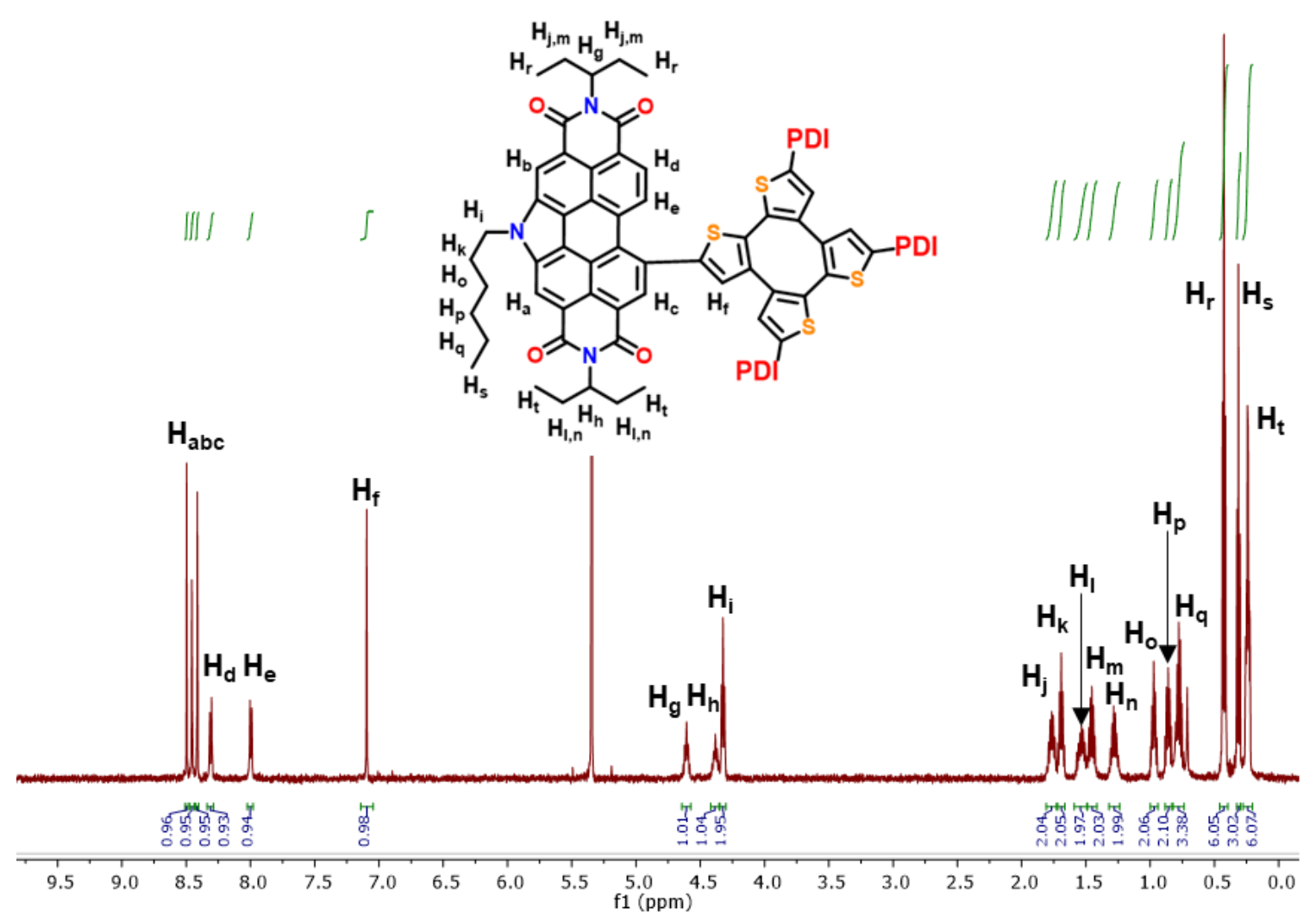

Figure S4. ${ }^{1} \mathrm{H}$ NMR spectrum of $\mathrm{Th}_{4} \mathrm{PDI}_{4}\left(600 \mathrm{MHz}\right.$, tetrachloroethane- $\left.\mathrm{d}_{2}, 120^{\circ} \mathrm{C}\right)$. 


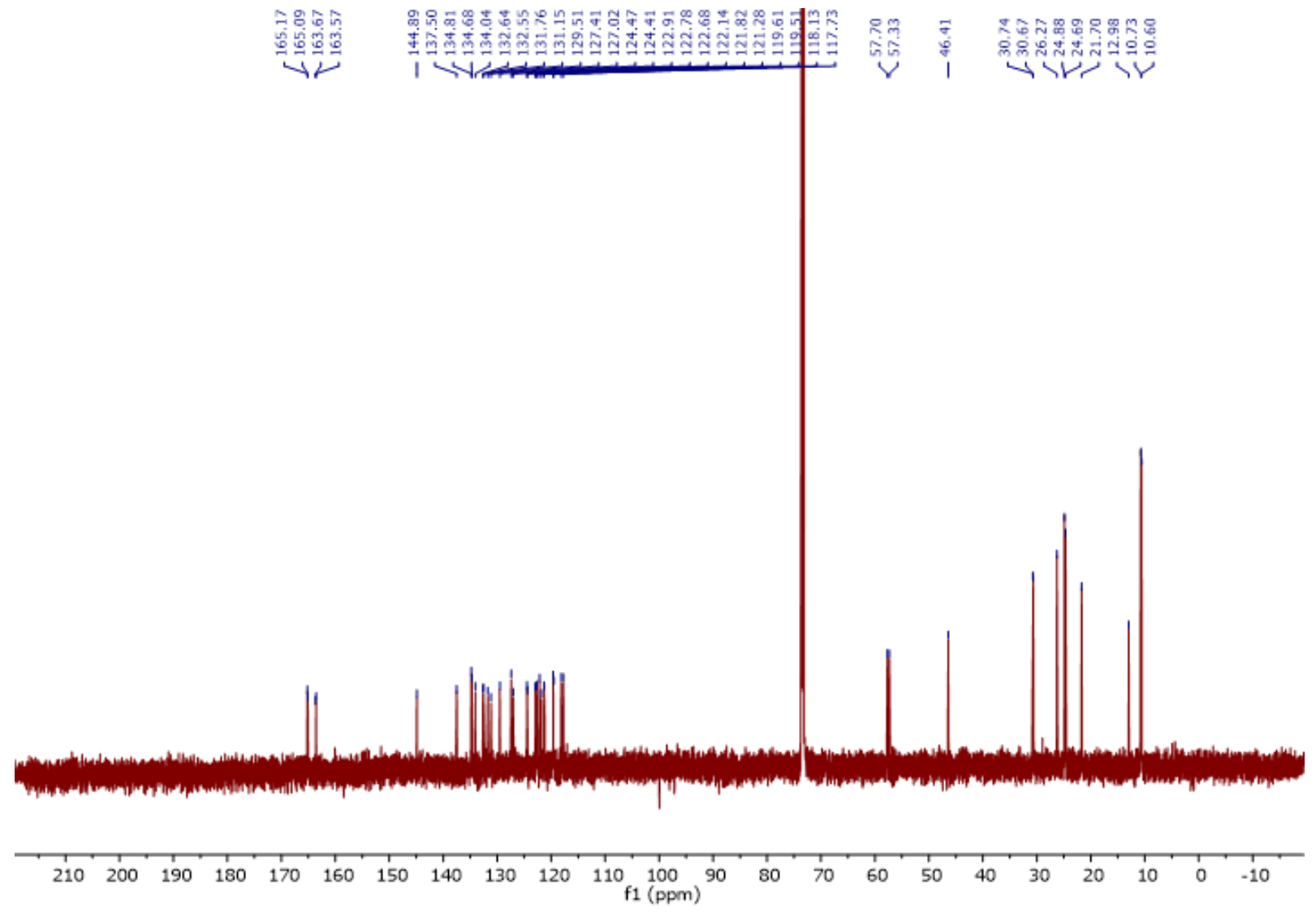

Figure S5. ${ }^{13} \mathrm{C}$ NMR spectrum of $\mathrm{Th}_{4} \mathrm{PDI}_{4}\left(151 \mathrm{MHz}\right.$, tetrachloroethane- $\left.\mathrm{d}_{2}, 120{ }^{\circ} \mathrm{C}\right)$.

\section{$1 \mathrm{mg} / \mathrm{mL}$}
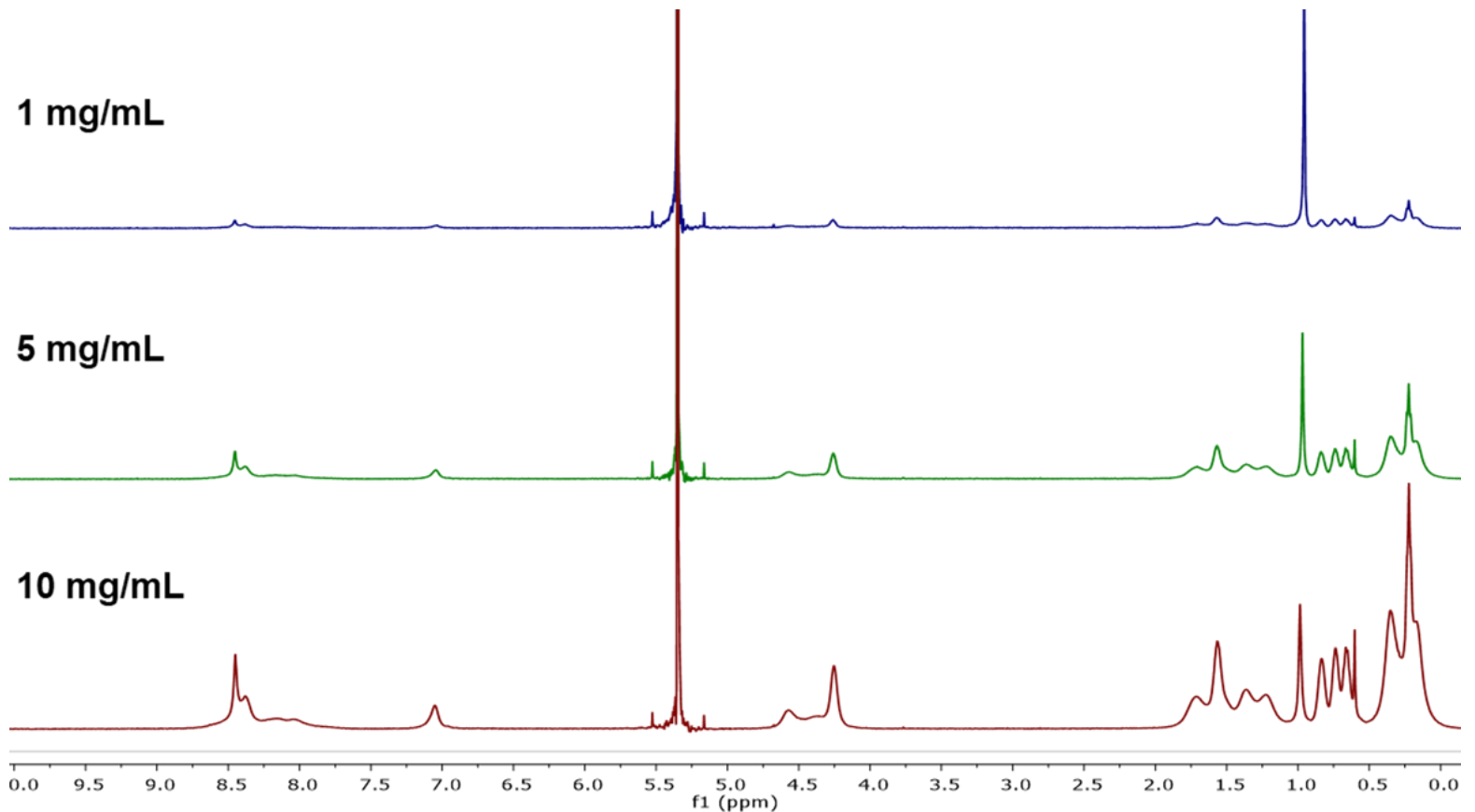

Figure S6. Concentration-dependent ${ }^{1} \mathrm{H}$ NMR spectra of $\mathrm{Th}_{4} \mathrm{PDI}_{4}\left(600 \mathrm{MHz}\right.$, tetrachloroethane-d $\mathrm{d}_{2}$, $\left.20^{\circ} \mathrm{C}\right)$. 


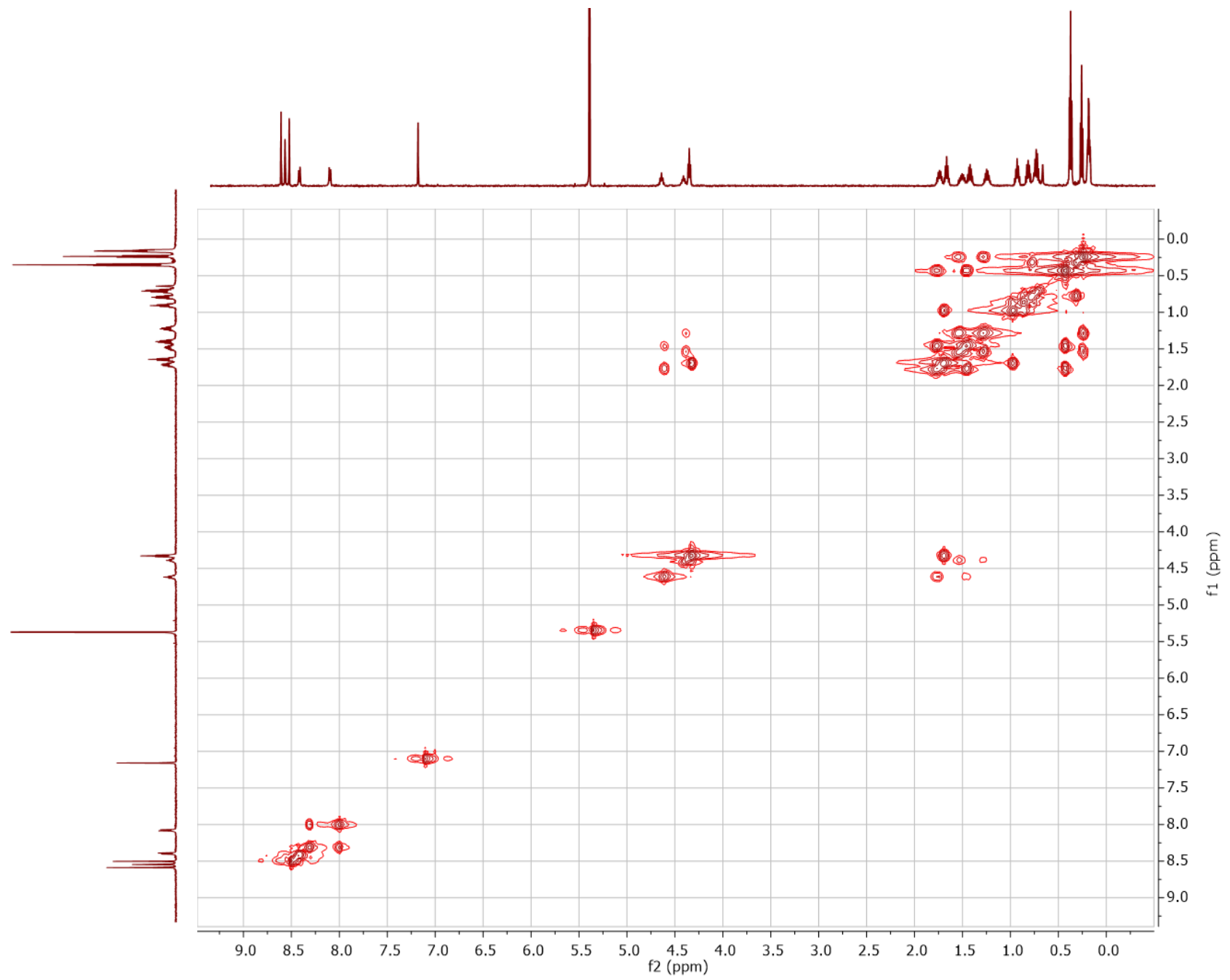

Figure S7. ${ }^{1} \mathrm{H}-{ }^{1} \mathrm{H}$ COSY spectrum of $\mathrm{Th}_{4} \mathrm{PDI}_{4}\left(600 \mathrm{MHz}\right.$, tetrachloroethane- $\left.\mathrm{d}_{2}, 120{ }^{\circ} \mathrm{C}\right)$. 


\section{HR-MALDI-TOF}

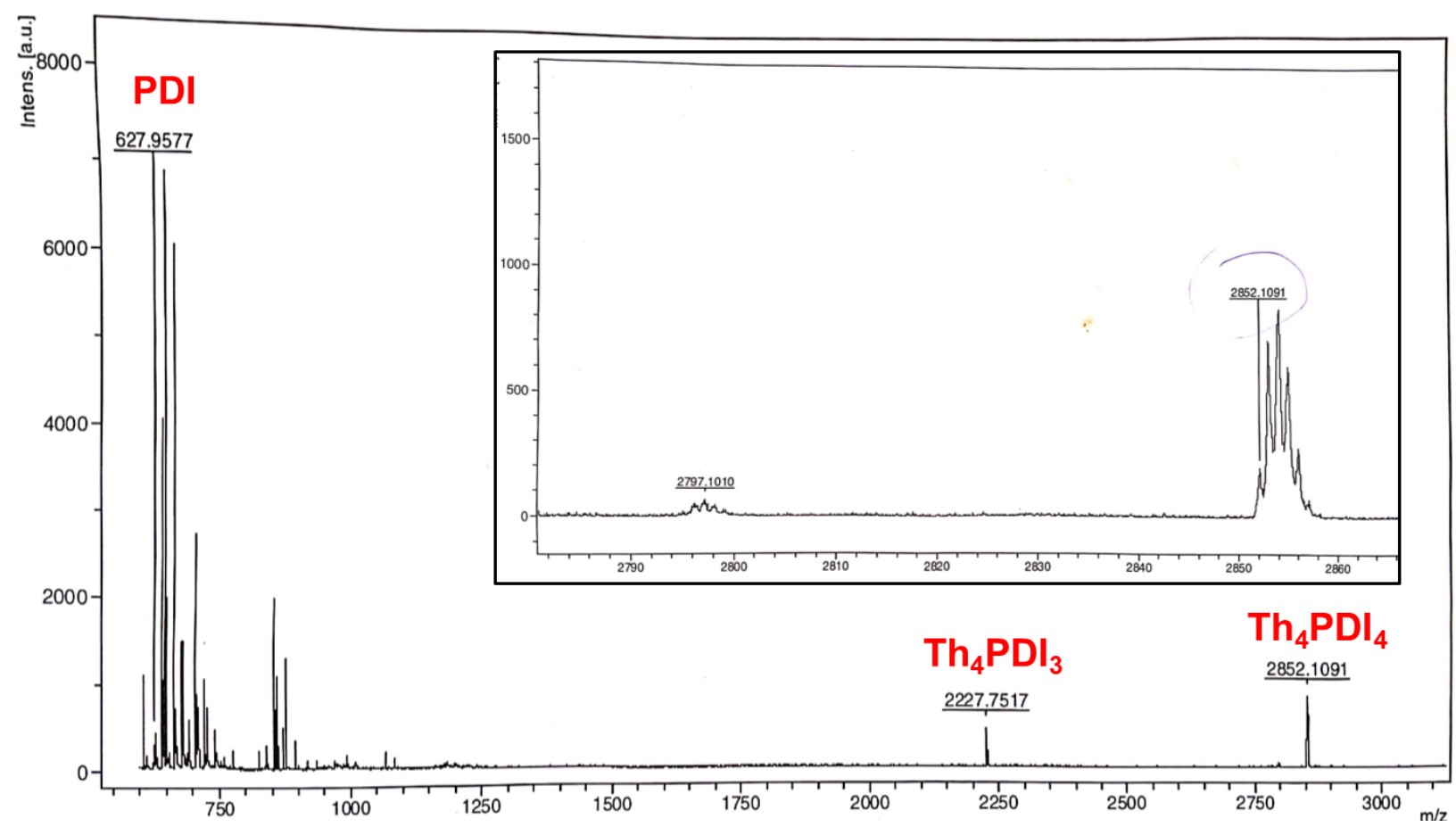

Figure S8. HR-MALDI-TOF mass spectrum showing incomplete substitution of $\mathrm{Th}_{4}$ core.

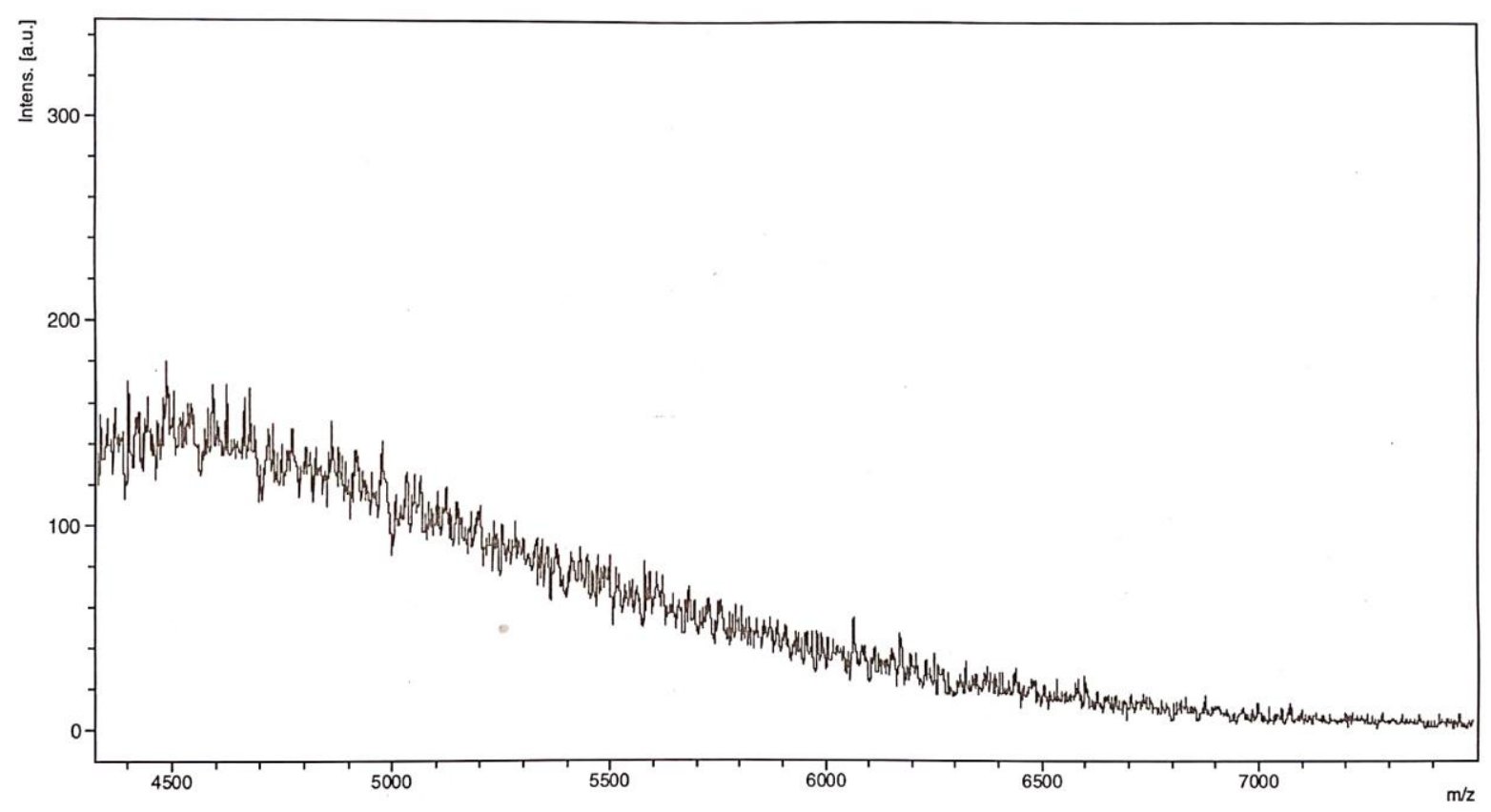

Figure S9. HR-MALDI-TOF mass spectrum showing no formation of $\mathrm{Th}_{4} \mathrm{PDI}_{8}$. 


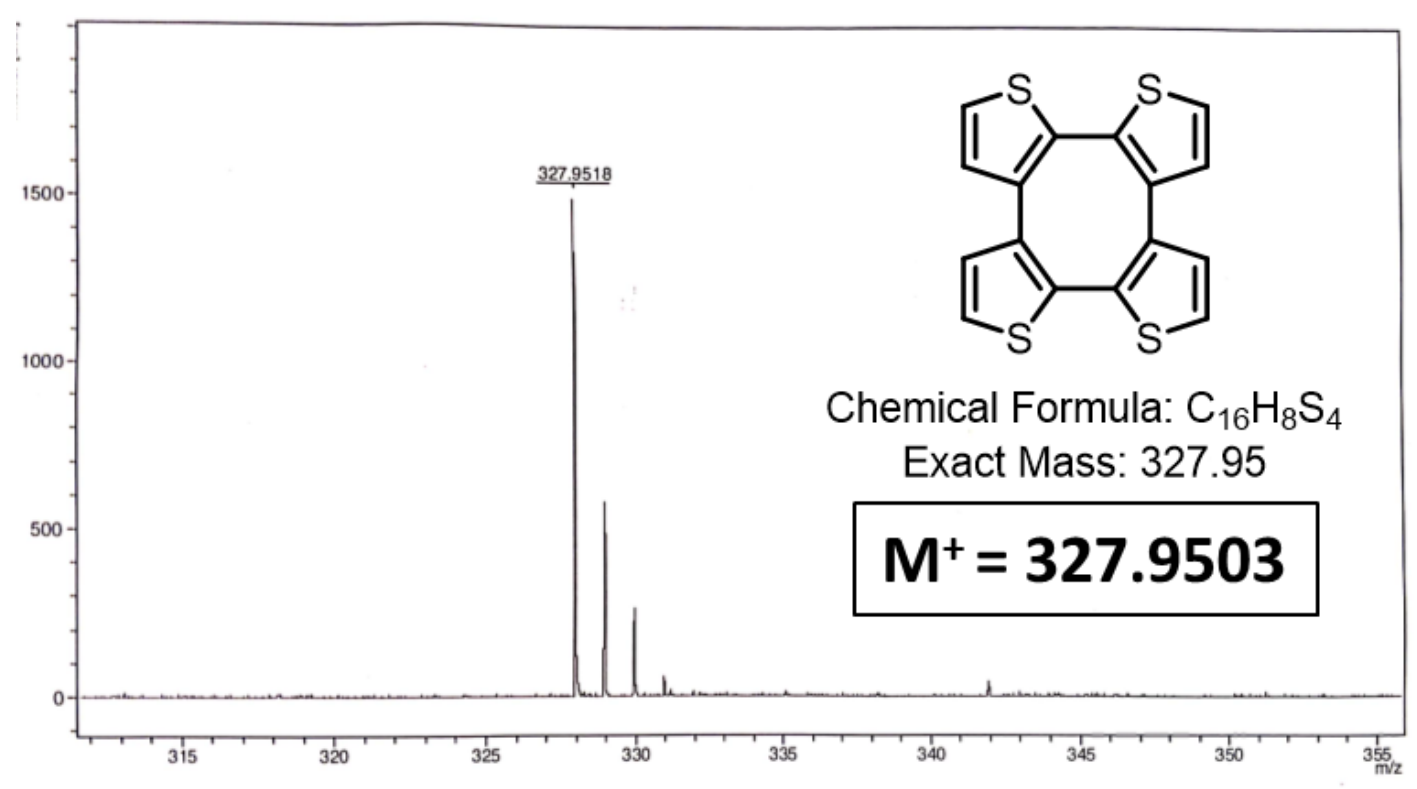

Figure S10. HR-MALDI-TOF mass spectrum of $\mathrm{Th}_{4}$.

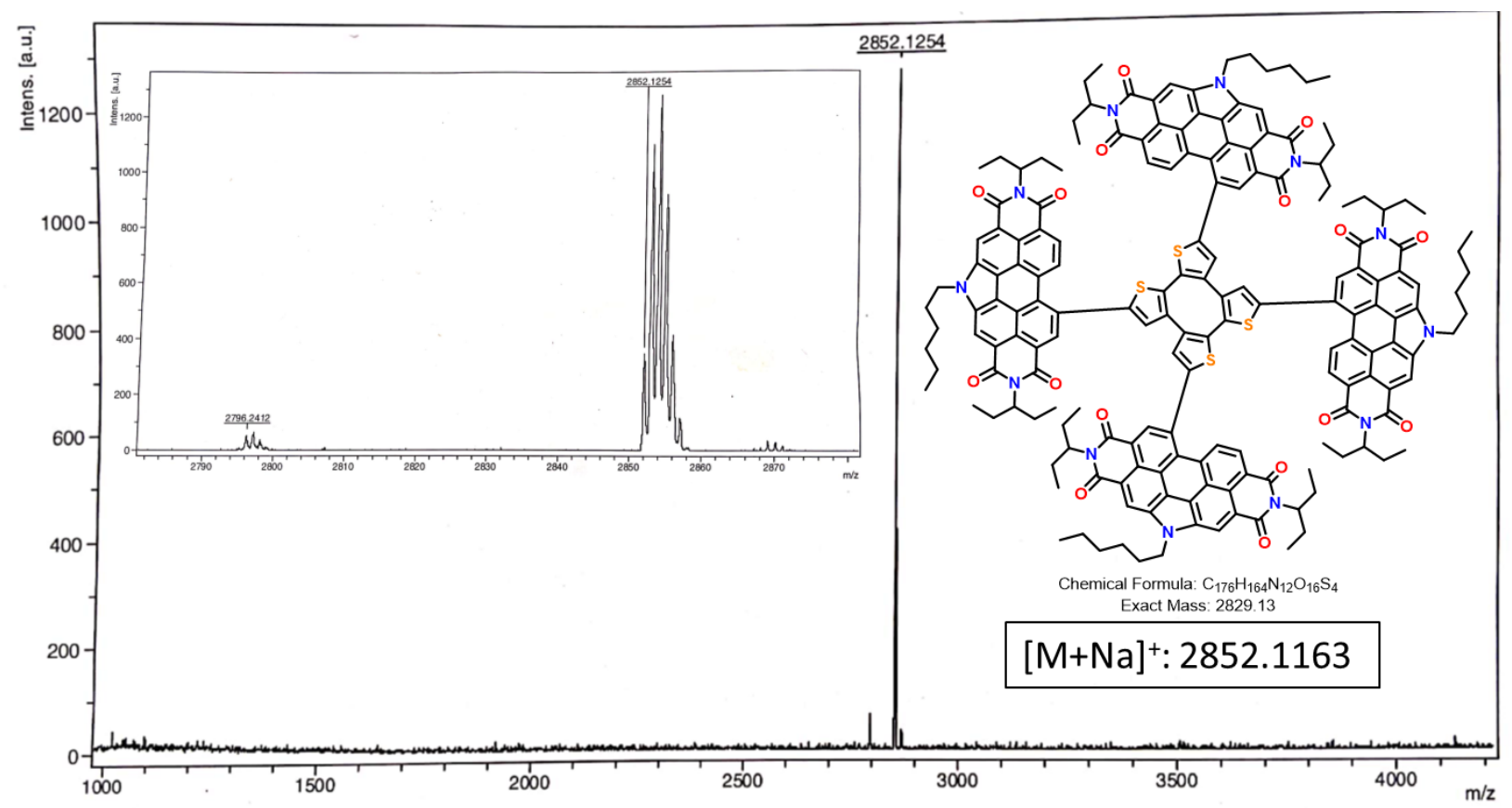

Figure S11. HR-MALDI-TOF mass spectrum of purified $\mathrm{Th}_{4} \mathrm{PDI}_{4}$. 


\section{Elemental Analysis \& Thermal Properties}

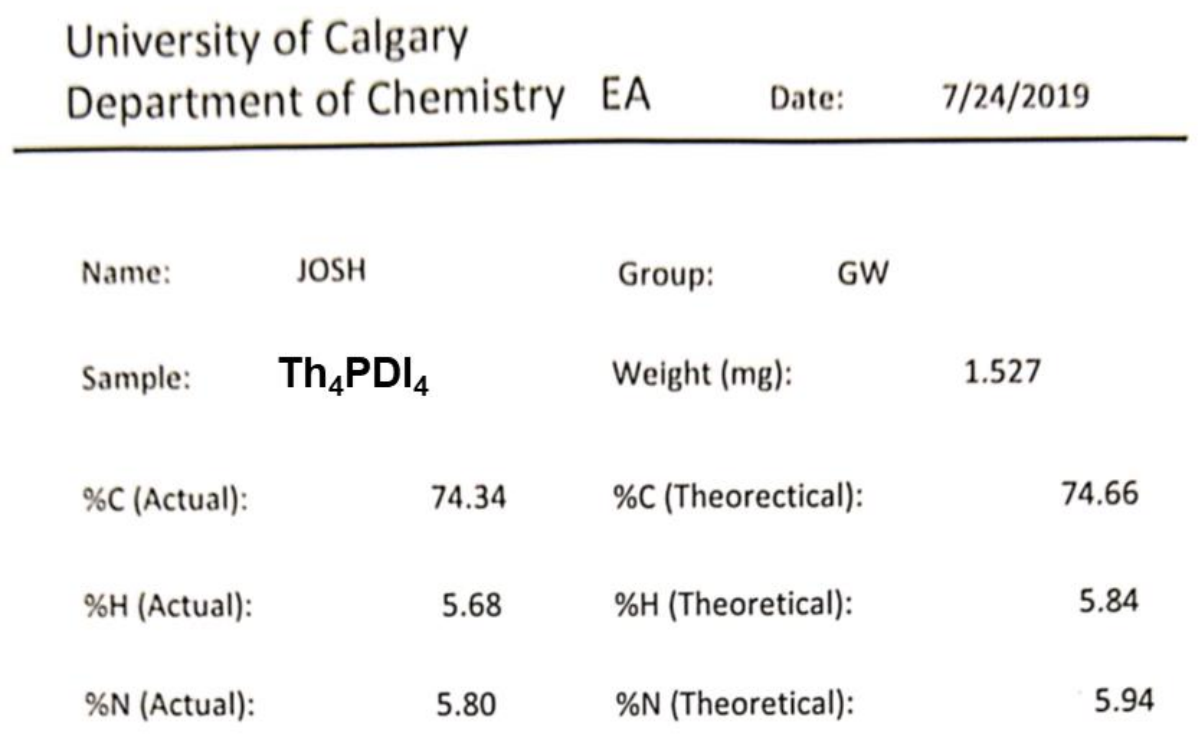

Figure S12. CHN elemental analysis of $\mathrm{Th}_{4} \mathrm{PDI}_{4}$.
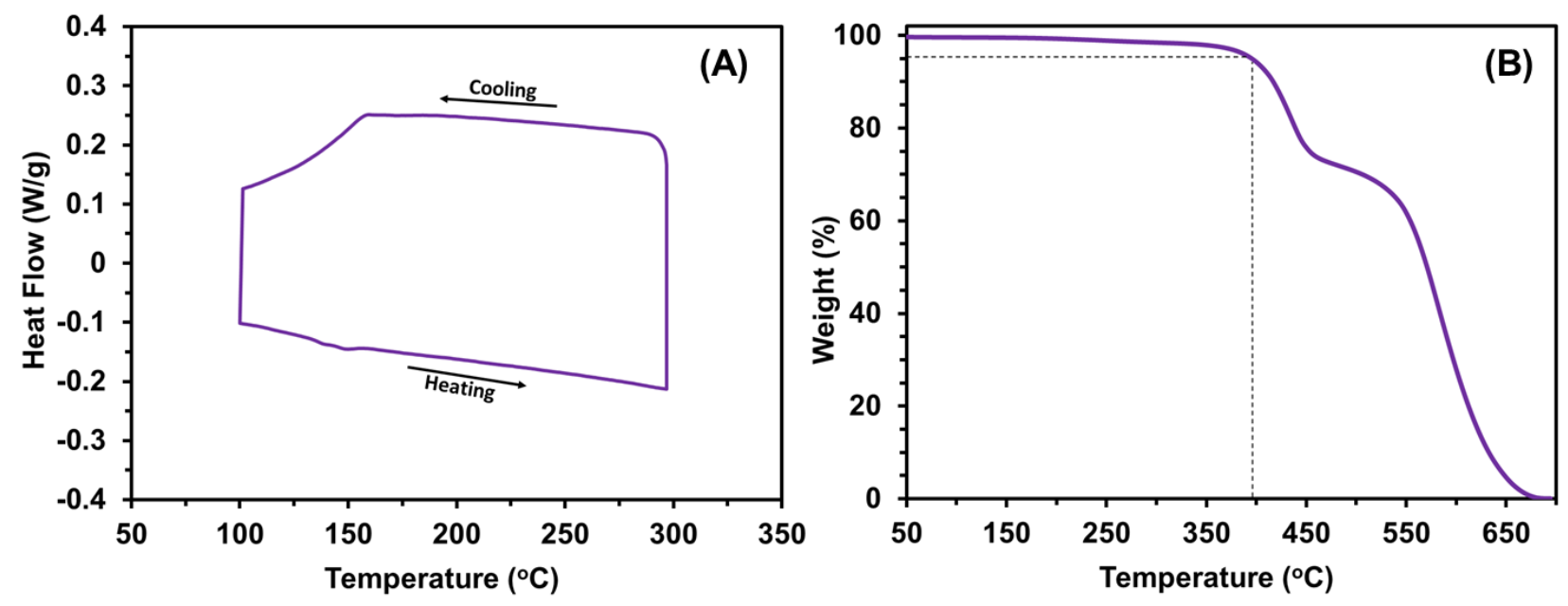

Figure S13. Differential scan calorimetry (A) and thermal gravimetric analysis (B) profiles of $\mathrm{Th}_{4} \mathrm{PDI}_{4}$. All features observed in the DSC trace of $\mathrm{Th}_{4} \mathrm{PDI}_{4}$ near $150{ }^{\circ} \mathrm{C}$ are artifacts of the instrument, as they were also observed in the blank. 


\section{UV-Visible Spectroscopy \& X-Ray Diffraction}

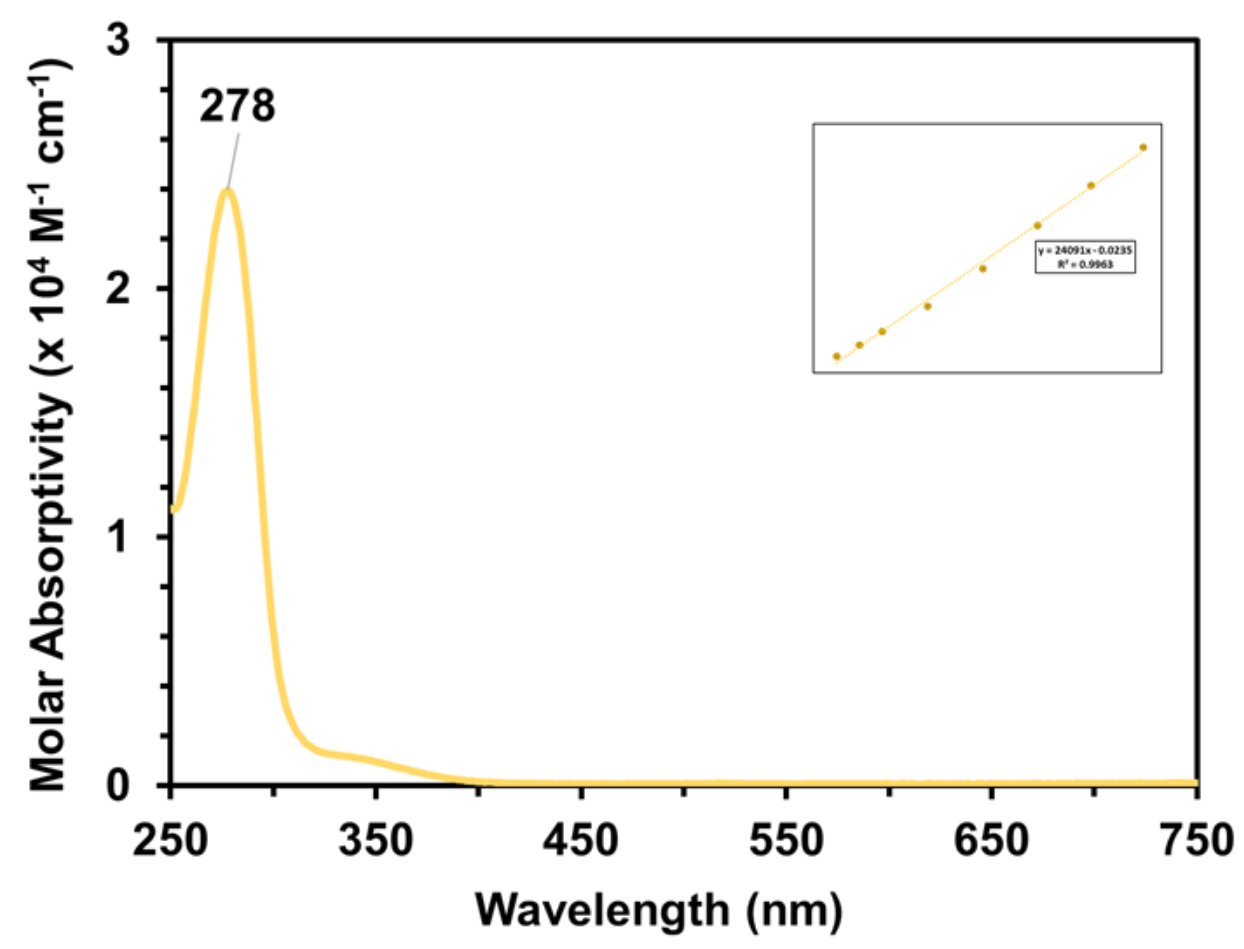

Figure S14. UV-visible absorption spectrum of $\mathrm{Th}_{4}$ in $\mathrm{CHCl}_{3}$, with calibration curve inset.

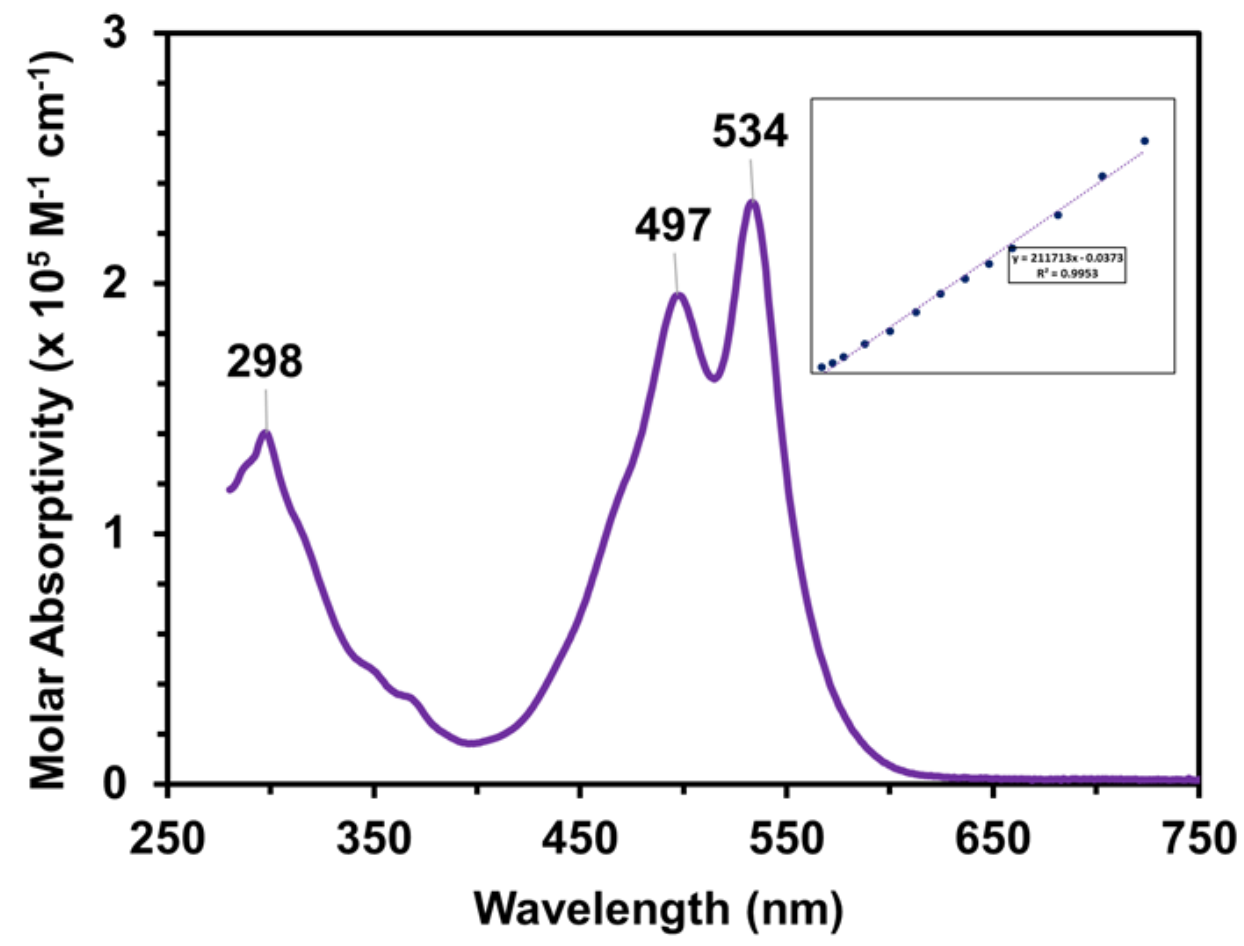

Figure S15. UV-visible absorption spectrum of $\mathrm{Th}_{4} \mathrm{PDI}_{4}$ in $\mathrm{CHCl}_{3}$, with calibration curve inset. 

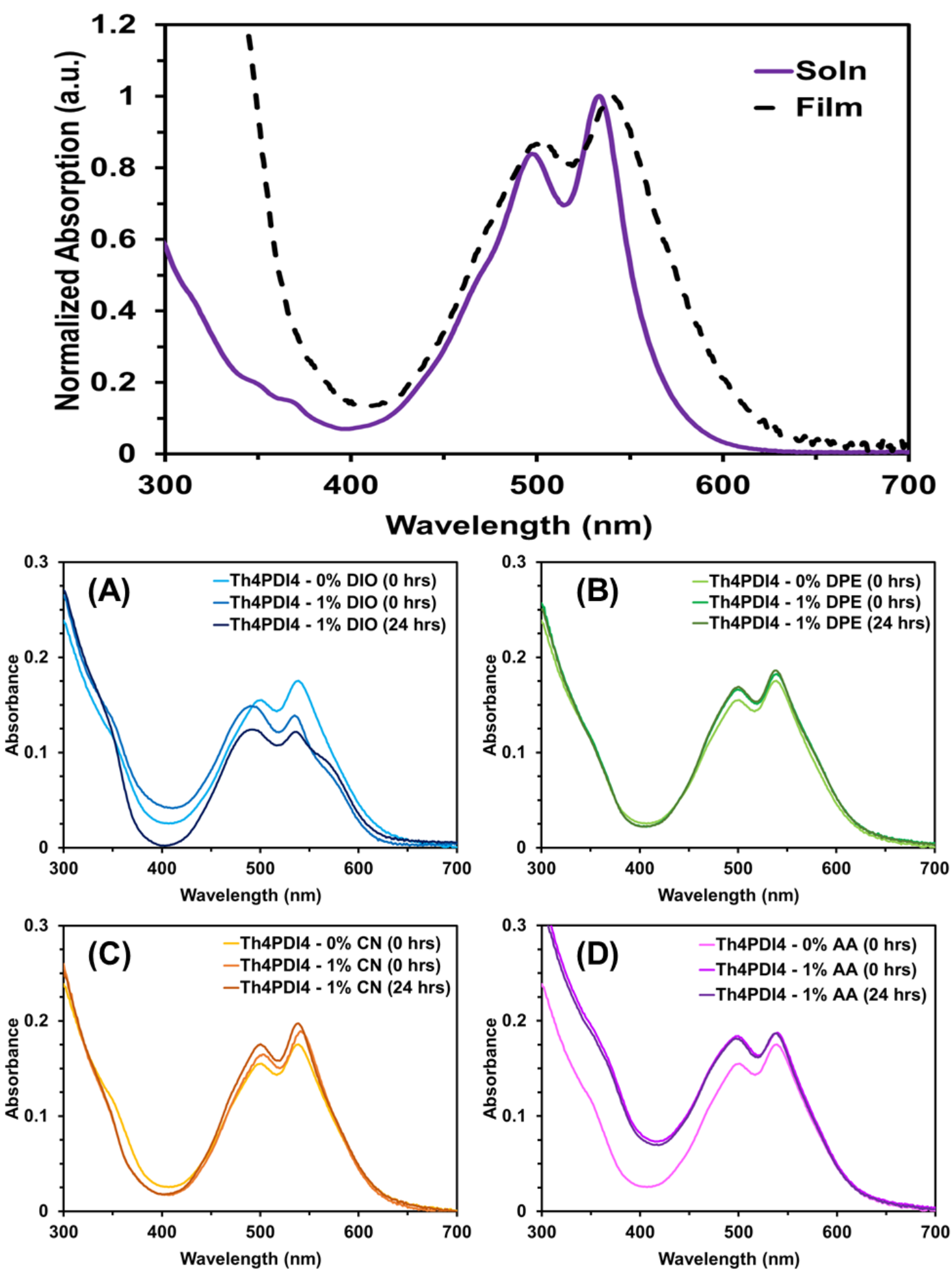

Figure S16. Directly comparing the UV-vis absorption spectra of $\mathrm{Th}_{4} \mathrm{PDI}_{4}$ in solution (o-DCB) and in thin film. The influence of 1,8-diiodooctane (A), diphenyl ether (B), chloronaphthalene (C), and $p$-anisaldehyde (D) on the thin film UV-vis absorption spectra of $\mathrm{Th}_{4} \mathrm{PDI}_{4}$ are also depicted. 

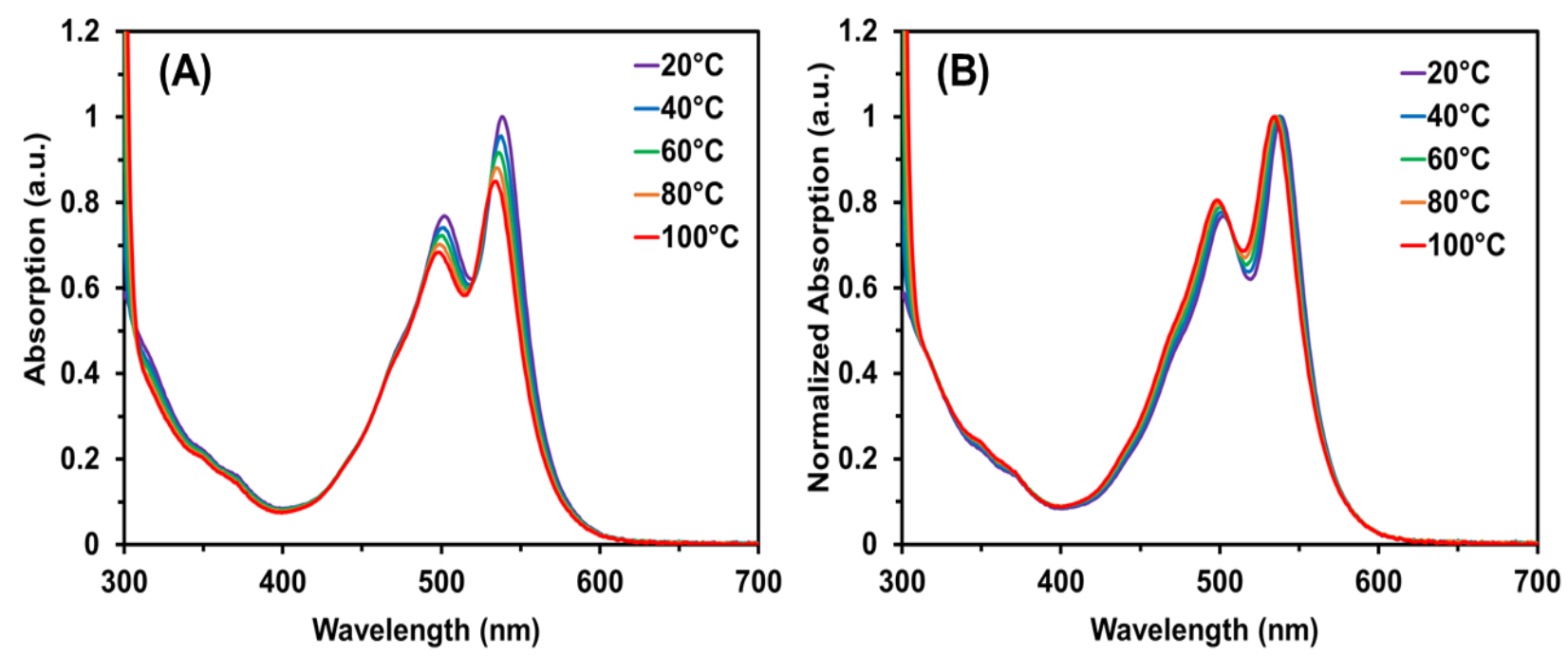

Figure S17. The raw (A) and normalized (B) variable-temperature UV-vis absorption spectra of $\mathrm{Th}_{4} \mathrm{PDI}_{4}\left(10^{-5} \mathrm{M}\right.$ in $\left.o-\mathrm{DCB}\right)$.

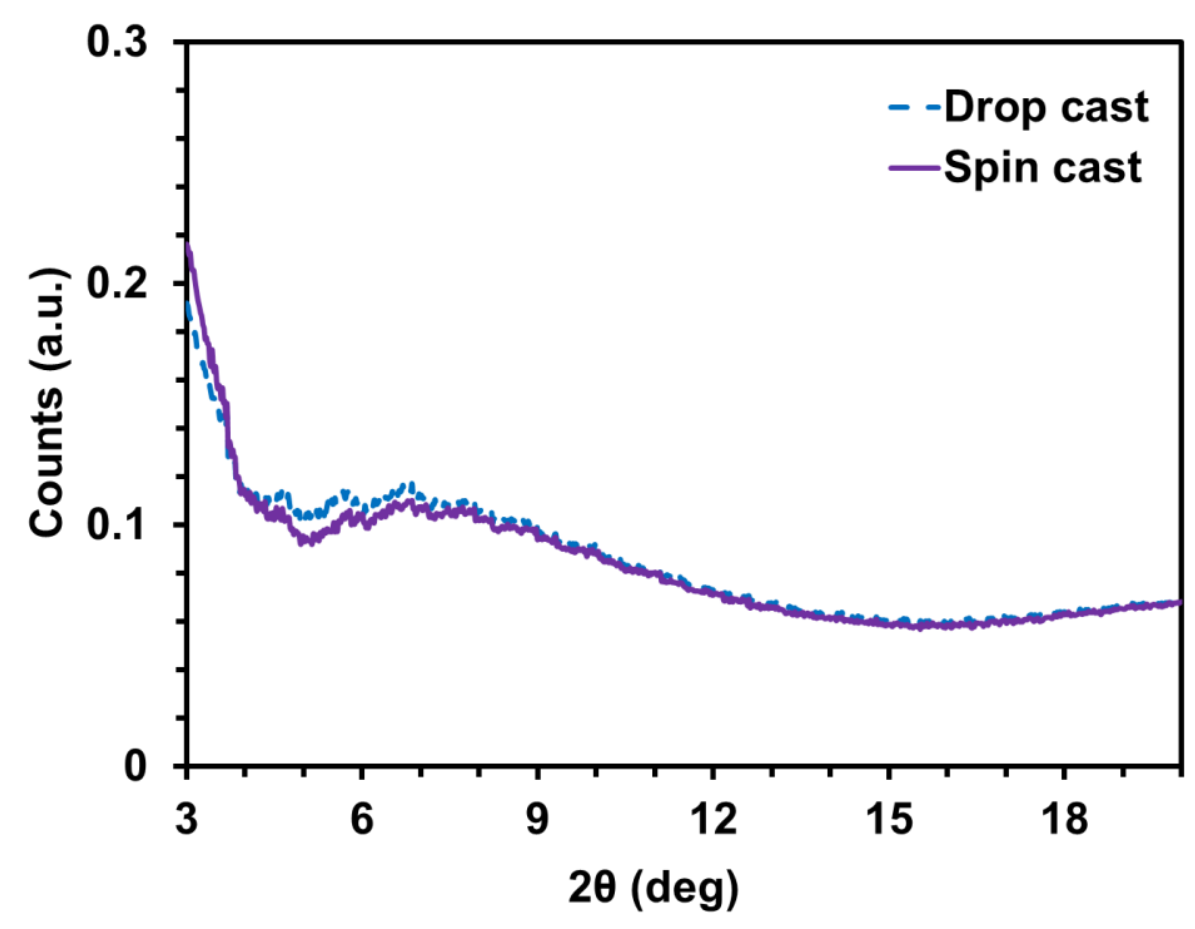

Figure S18. X-ray diffraction spectra of drop cast and spin cast $\mathrm{Th}_{4} \mathrm{PDI}_{4}$ thin films. 


\section{Voltammetry}

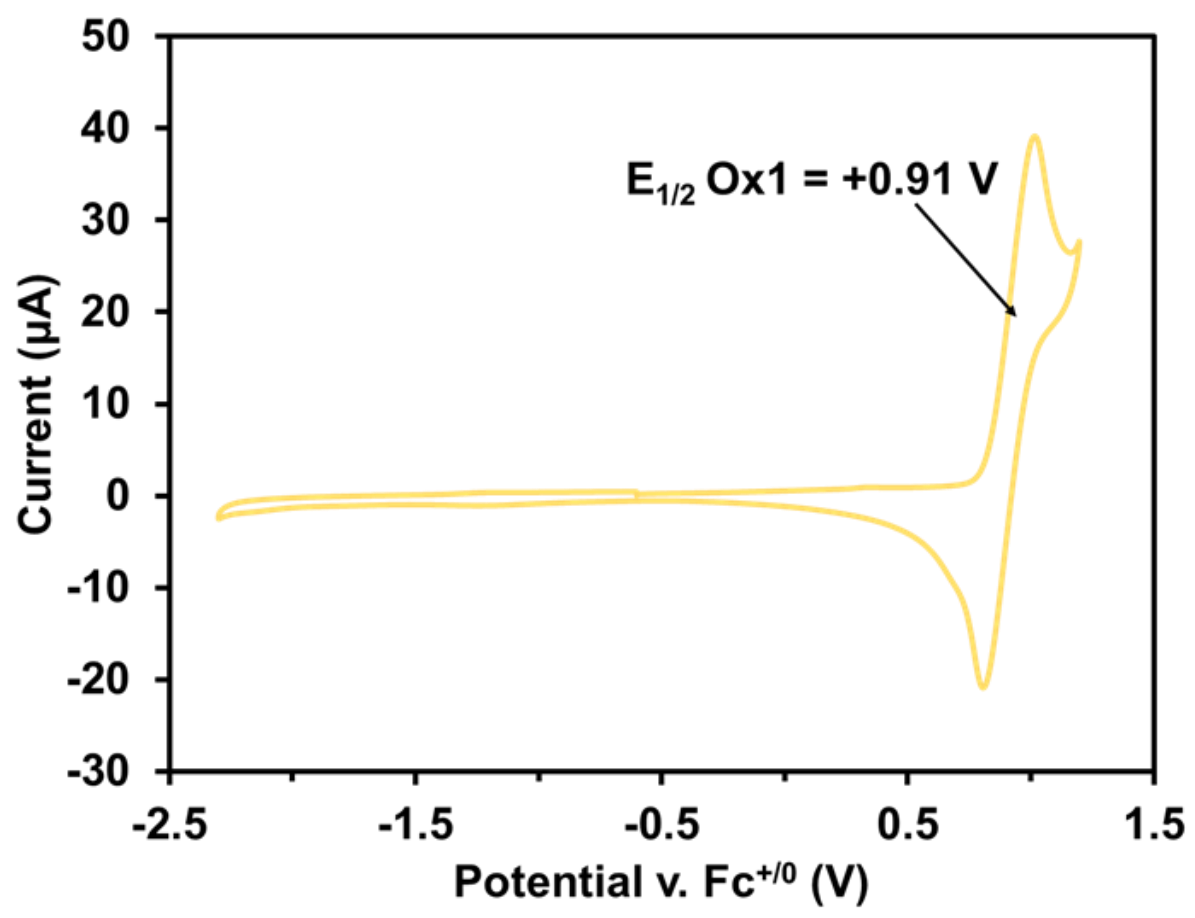

Figure S19. Cyclic voltammogram of $\mathrm{Th}_{4}(\sim 1 \mathrm{mM})$ in $\mathrm{CH}_{2} \mathrm{Cl}_{2}$, recorded at $100 \mathrm{mV} / \mathrm{s}$.
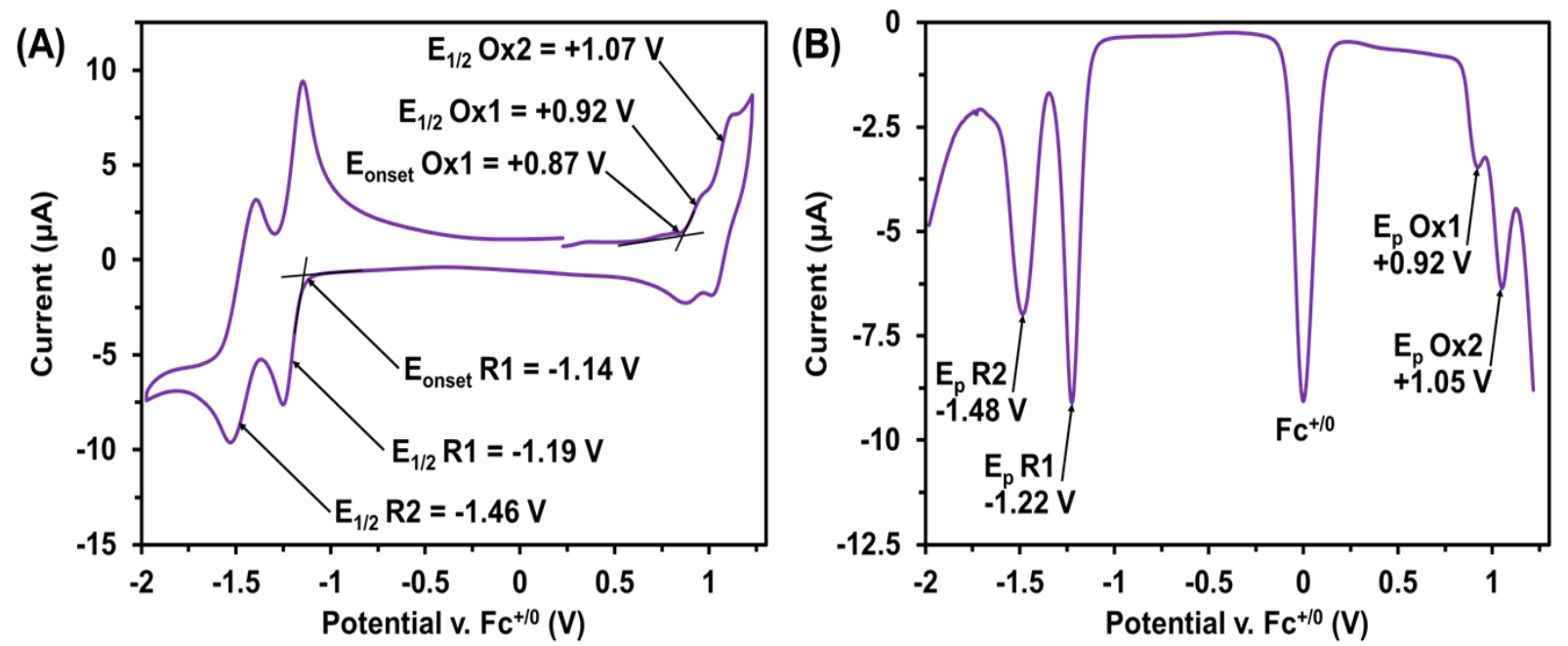

Figure S20. Cyclic voltammogram (A) and differential pulse voltammogram (B) of $\mathrm{Th}_{4} \mathrm{PDI}_{4}$ $(\sim 1 \mathrm{mM})$ in $\mathrm{CH}_{2} \mathrm{Cl}_{2}$, recorded at $100 \mathrm{mV} / \mathrm{s}$. 


\section{Density Functional Theory}
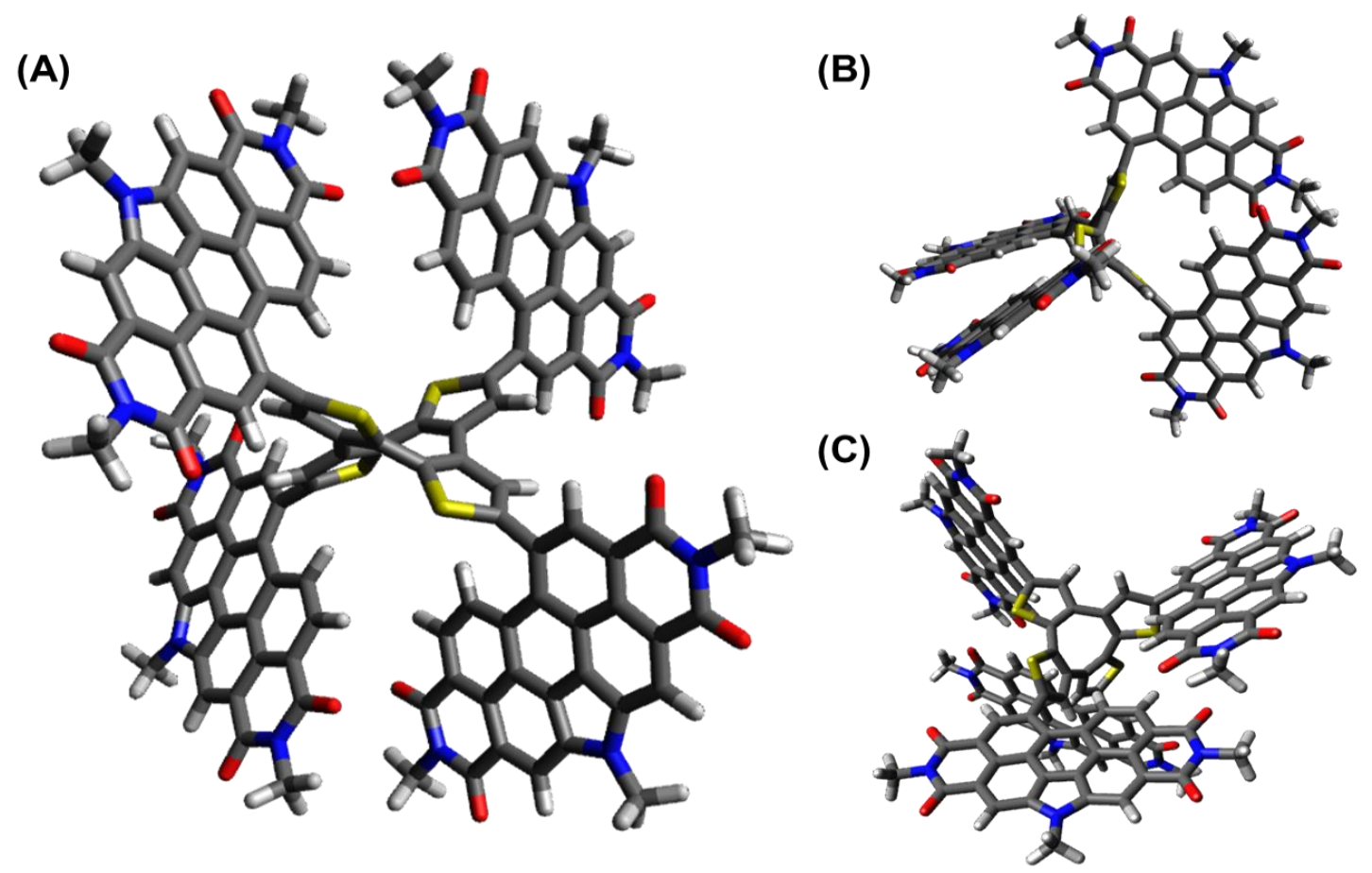

Figure S21. Optimized geometry of $\mathrm{Th}_{4} \mathrm{PDI}_{4}$ at B3LYP/6-31G(d,p) ground-state.

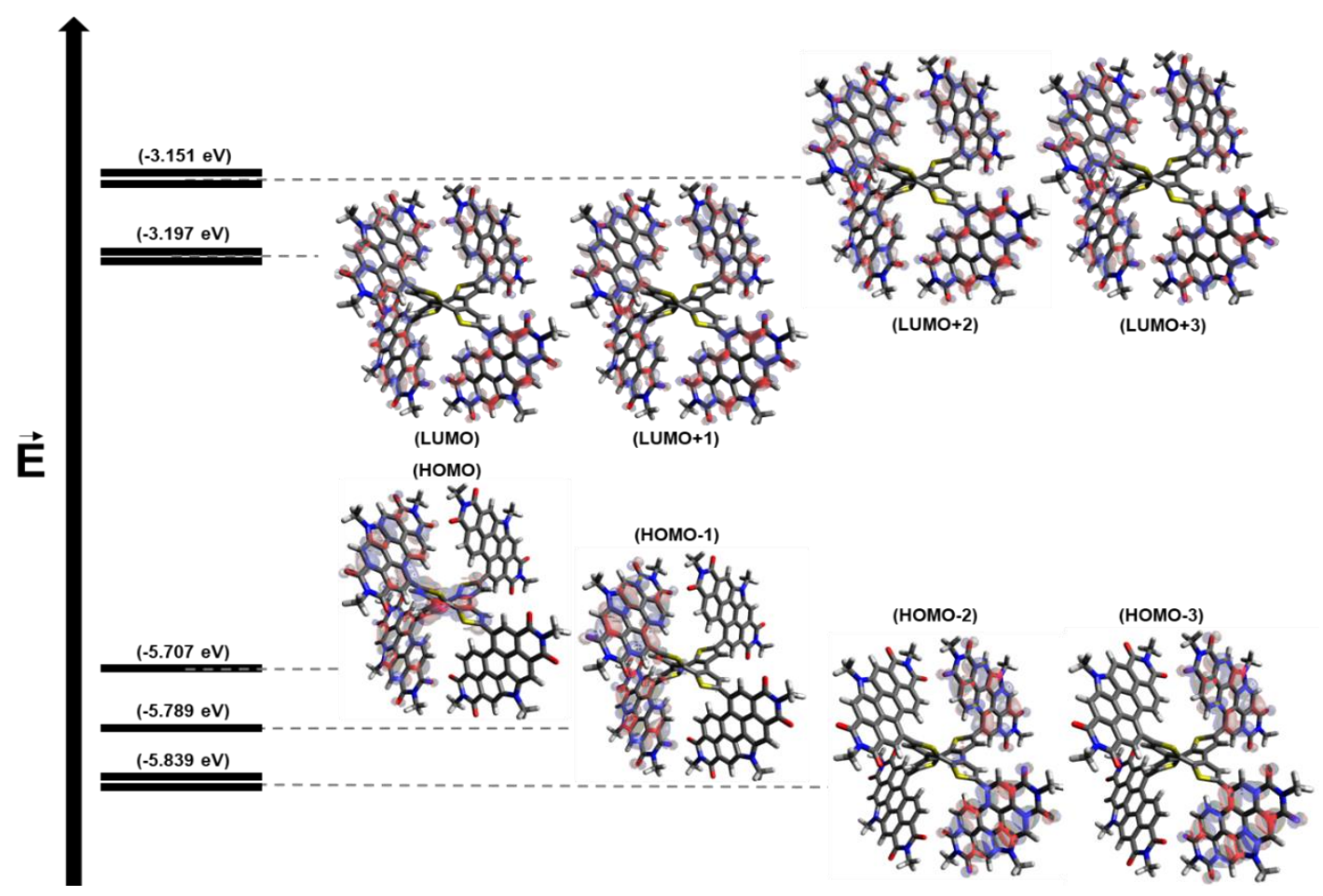

Figure S22. Molecular orbital diagram of $\mathrm{Th}_{4} \mathrm{PDI}_{4}$ at B3LYP/6-31G(d,p) ground-state. Depicted energy-levels represent DFT calculated values, rather than experimentally determined values. 


\section{OPV Device Optimization}

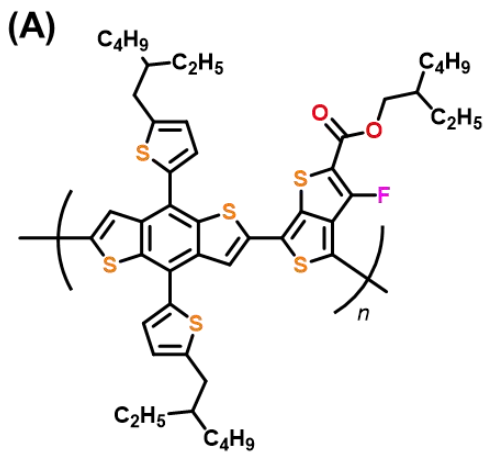

(B)

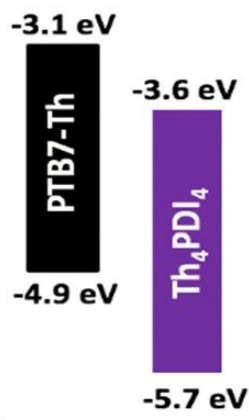

(C)

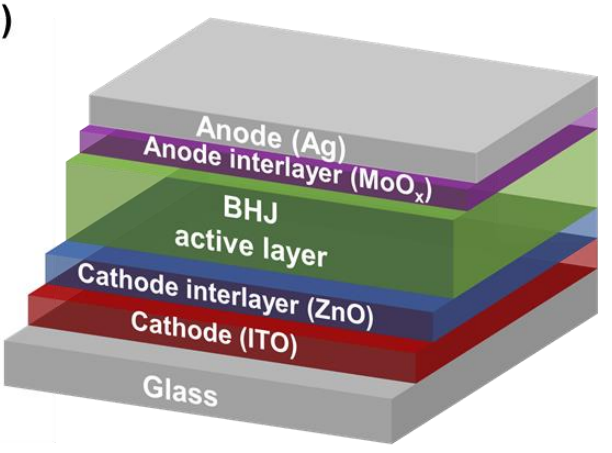

Figure S23. Chemical structure of PTB7-Th (A), CV determined energy levels (using E $_{1 / 2}$ ) for PTB7-Th and $\mathrm{Th}_{4} \mathrm{PDI}_{4}$ (B), and proof-of-concept OPV device architecture (C).

(A)

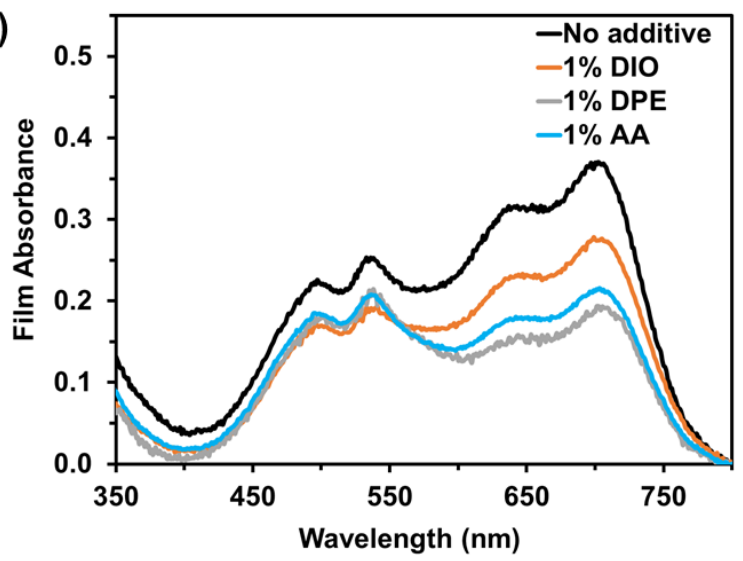

(B)

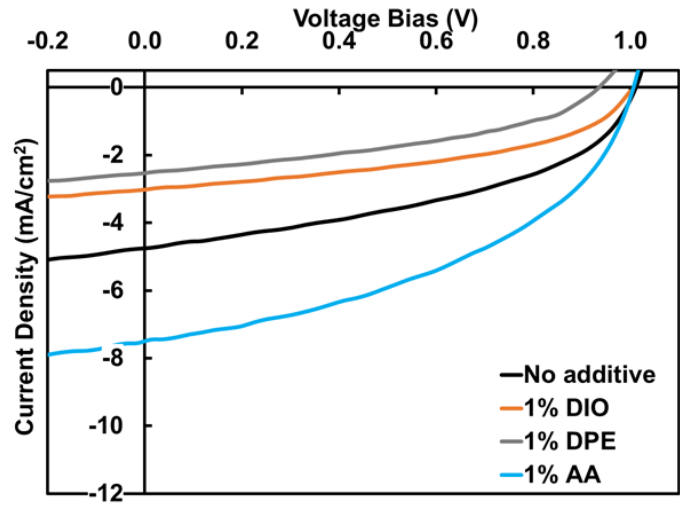

Figure S24. UV-visible spectra (A) and J-V curves (B) of $1: 1$ PTB7-Th:Th ${ }_{4} \mathrm{PDI}_{4}$ films and devices, respectively, spin-coated from $o$-DCB containing 0 or $1 \%(\mathrm{v} / \mathrm{v})$ of DIO, DPE or AA.

(A)

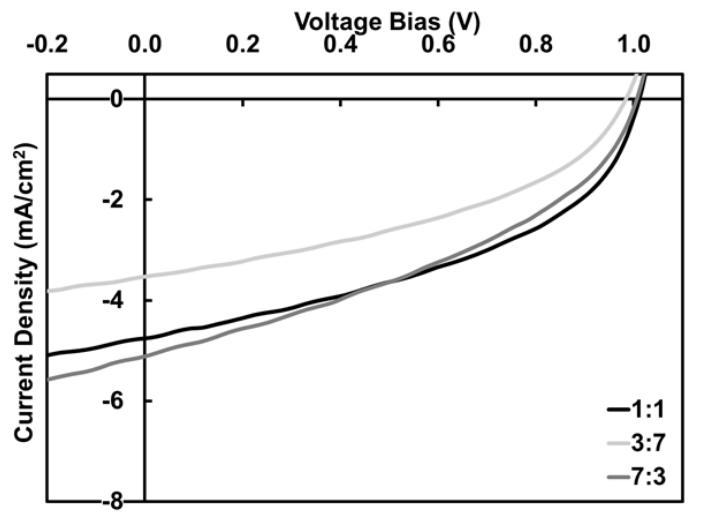

(B)

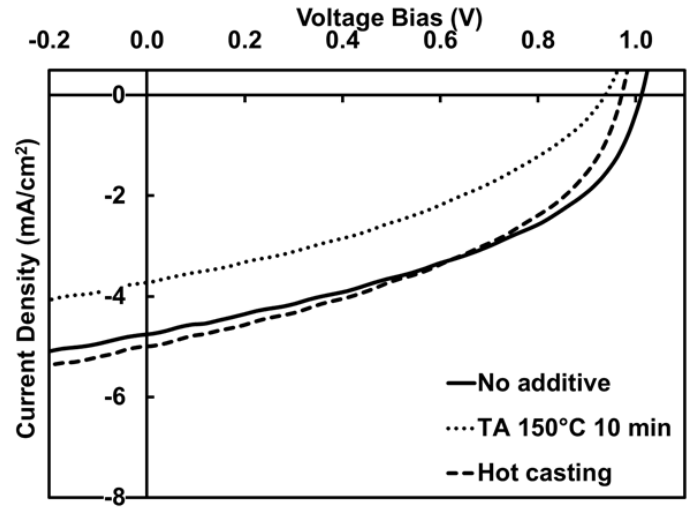

Figure S25. J-V curves of the PTB7-Th-Th ${ }_{4} \mathrm{PDI}_{4}$ devices spin-coated from $o$-DCB using different (A) donor:acceptor weight ratio and (B) different processing. 


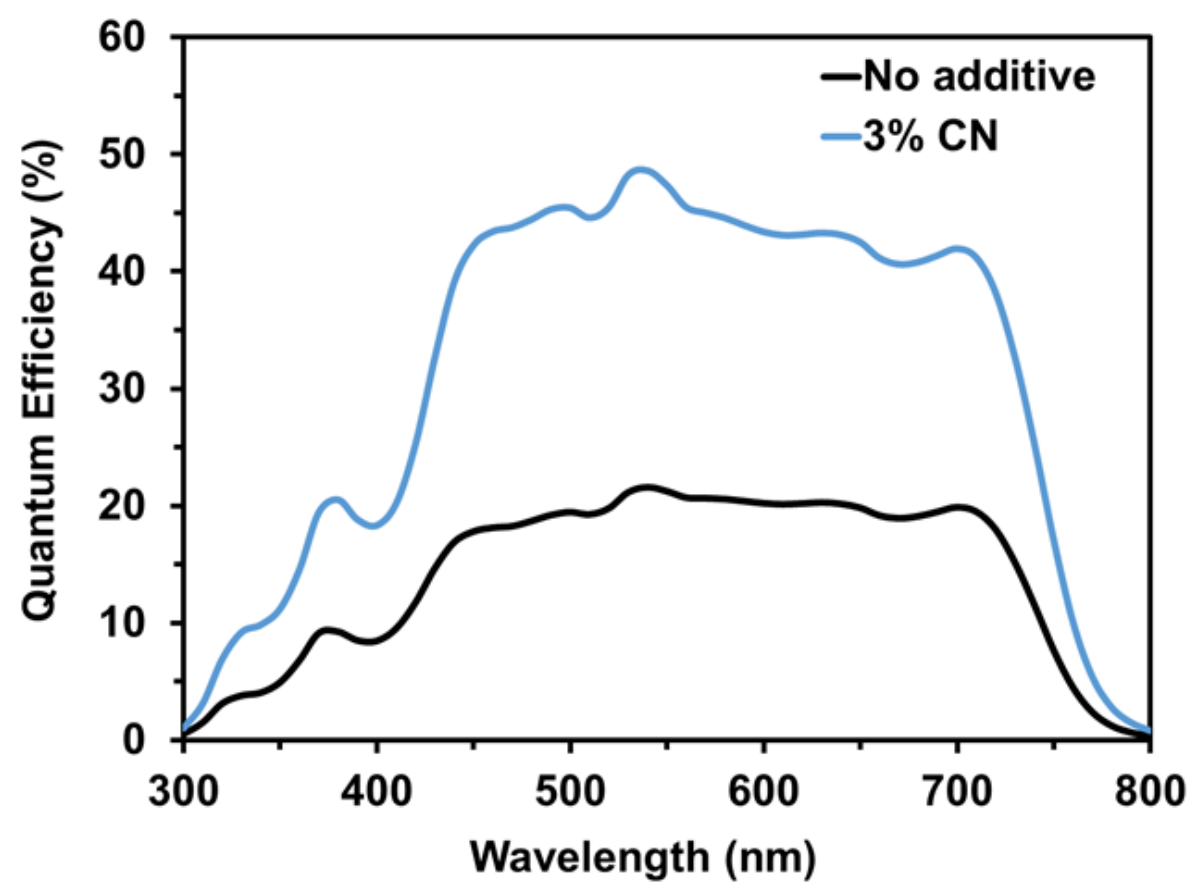

Figure S26. External quantum efficiency of 1:1 PTB7-Th:Th4 $\mathrm{PDI}_{4}$ films spin-coated from $o$-DCB containing 0 or $3 \%(\mathrm{v} / \mathrm{v}) \mathrm{CN}$.

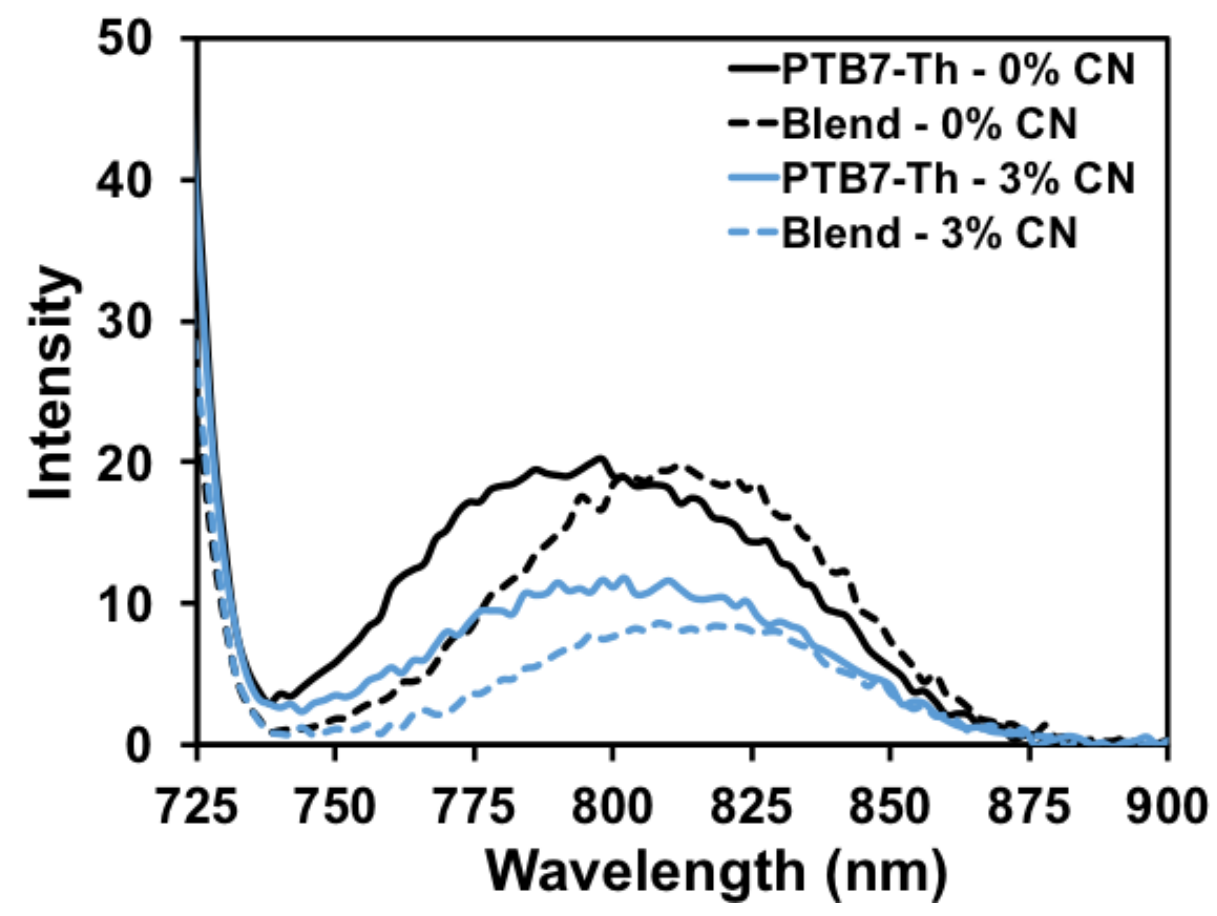

Figure S27. Photoluminescence spectra of PTB7-Th and of 1:1 PTB7-Th:Th ${ }_{4} \mathrm{PDI}_{4}$ films spincoated from $o$-DCB containing 0 or $3 \%(\mathrm{v} / \mathrm{v}) \mathrm{CN}$. The excitation wavelength is $705 \mathrm{~nm}$. 

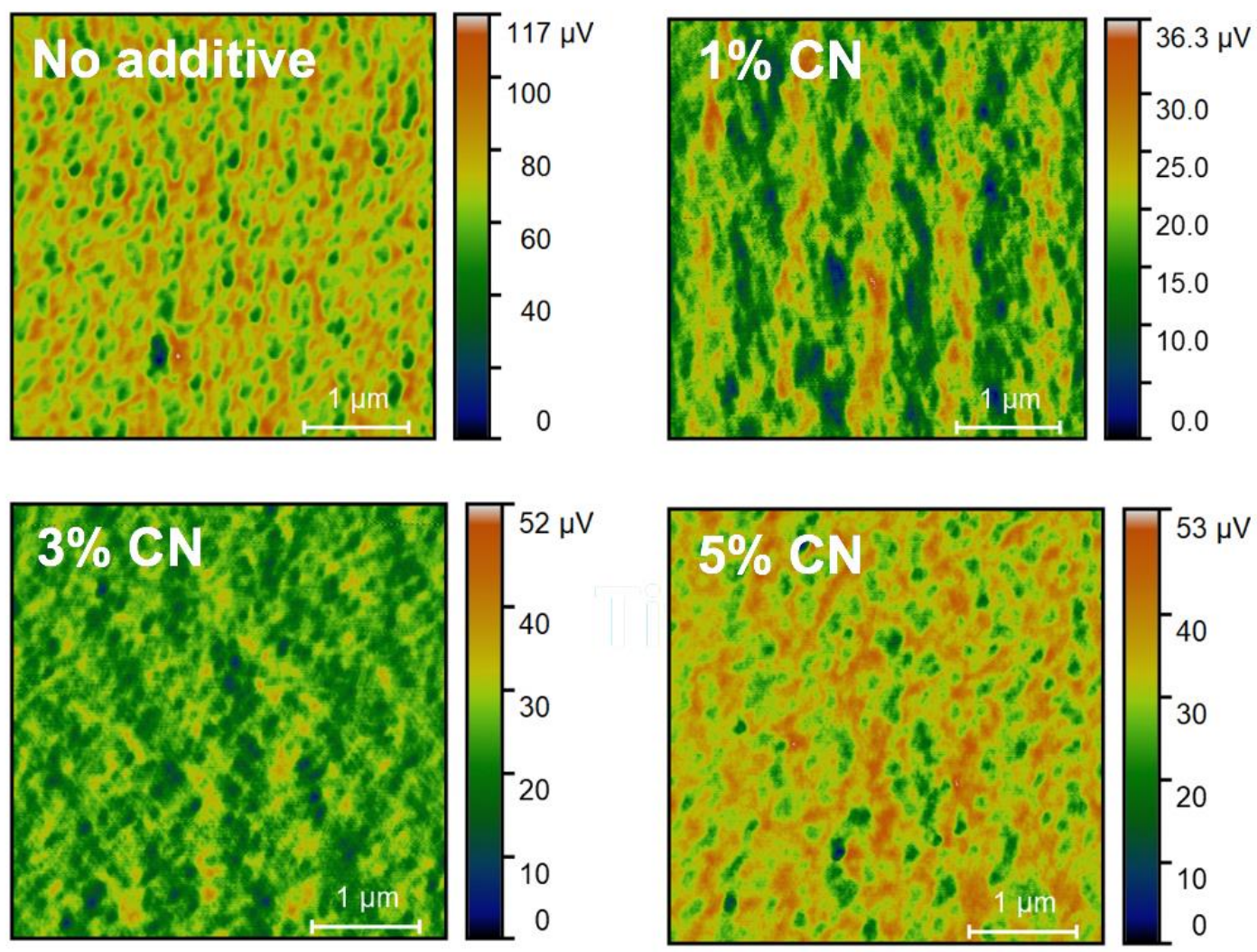

Figure S28. AFM phase images of the 1:1 PTB7-Th:Th ${ }_{4} \mathrm{PDI}_{4}$ films spin-coated from $o$-DCB containing $0,1,3$ or $5 \%(\mathrm{v} / \mathrm{v}) \mathrm{CN}$.
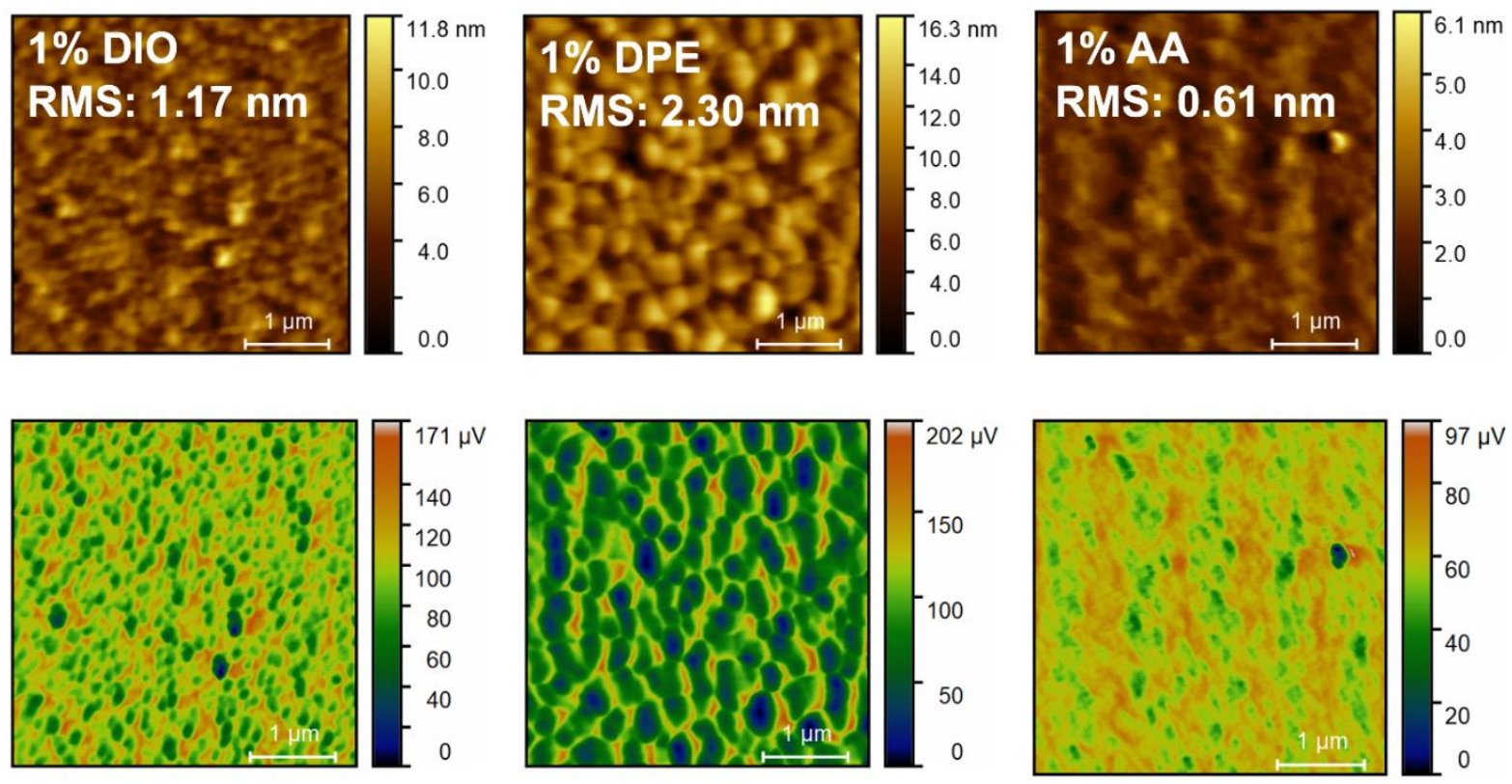

Figure S29. AFM height (top) and phase (bottom) images of the PTB7-Th:Th4 $\mathrm{PDI}_{4}$ devices spincoated from $o$-DCB using different processing conditions. 

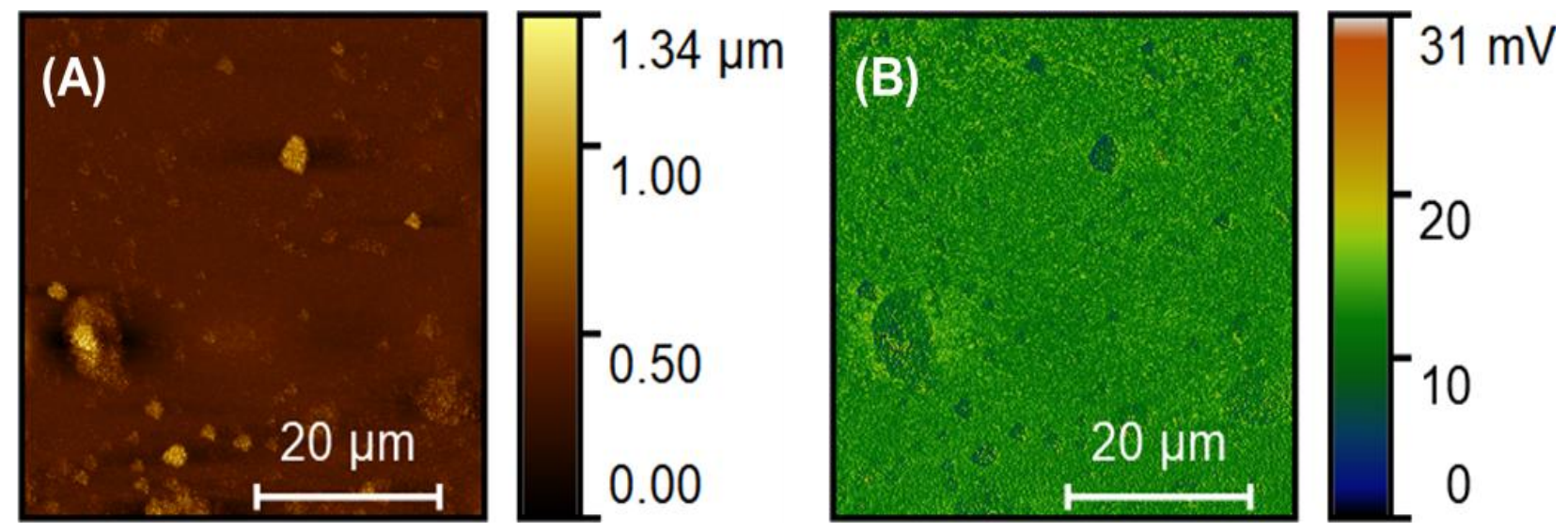

Figure S30. AFM height (A) and phase (B) images of the PTB7-Th:Th ${ }_{4} \mathrm{PDI}_{4}$ devices spin-coated from $o$-DCB using $3 \%(\mathrm{v} / \mathrm{v}) \mathrm{CN}$. The calculated film RMS was $74 \mathrm{~nm}$.

Table S1. OPV data of PTB7-Th:Th4 ${ }_{4} \mathrm{PDI}_{4}$ blends spin-coated from $o$-DCB with different additives. Best PCE results are highlighted in bold. Averages are in italics.

\begin{tabular}{|c|c|c|c|c|}
\hline Active layer & $V_{\text {oc }}(\mathrm{V})$ & $\mathrm{J}_{\mathrm{sc}}\left(\mathrm{mA} / \mathrm{cm}^{2}\right)$ & FF (\%) & PCE (\%) \\
\hline \multicolumn{5}{|c|}{$\begin{array}{l}o-\mathrm{DCB}, 15 \mathrm{mg} / \mathrm{mL} \text {, } \\
\text { 1:1 ratio }\end{array}$} \\
\hline \multirow[t]{8}{*}{ No additive } & 1.00 & 4.3 & 40 & 1.72 \\
\hline & 1.02 & 5.1 & 42 & 2.20 \\
\hline & 1.02 & 4.8 & 43 & 2.10 \\
\hline & 1.01 & 4.7 & 44 & 2.10 \\
\hline & 1.03 & 3.8 & 43 & 1.68 \\
\hline & 1.01 & 4.3 & 40 & 1.72 \\
\hline & 1.01 & 4.4 & 39 & 1.73 \\
\hline & 1.01 & 4.5 & 42 & 1.89 \\
\hline \multirow[t]{7}{*}{$1 \%(\mathrm{v} / \mathrm{v}) \mathrm{DIO}$} & 1.01 & 3.0 & 46 & 1.39 \\
\hline & 1.01 & 3.0 & 45 & 1.36 \\
\hline & 1.03 & 2.9 & 44 & 1.31 \\
\hline & 1.01 & 2.9 & 45 & 1.30 \\
\hline & 1.04 & 3.6 & 45 & 1.67 \\
\hline & 1.02 & 3.6 & 44 & 1.63 \\
\hline & 1.02 & 3.2 & 45 & 1.44 \\
\hline \multirow[t]{4}{*}{$1 \%(\mathrm{v} / \mathrm{v}) \mathrm{DPE}$} & 0.97 & 2.6 & 40 & 1.02 \\
\hline & 0.93 & 2.5 & 40 & 0.95 \\
\hline & 0.91 & 2.5 & 43 & 0.99 \\
\hline & 0.94 & 2.6 & 41 & 0.99 \\
\hline \multirow[t]{5}{*}{$1 \%(\mathrm{v} / \mathrm{v}) \mathrm{AA}$} & 0.98 & 6.9 & 40 & 2.72 \\
\hline & 1.00 & 8.1 & 44 & 3.53 \\
\hline & 1.00 & 7.5 & 44 & 3.32 \\
\hline & 1.00 & 7.4 & 44 & 3.27 \\
\hline & 1.00 & 7.5 & 43 & 3.21 \\
\hline
\end{tabular}




\begin{tabular}{|c|c|c|c|c|}
\hline \multirow[t]{9}{*}{$1 \%(\mathrm{v} / \mathrm{v}) \mathrm{CN}$} & 0.97 & 9.6 & 37 & 3.51 \\
\hline & 0.97 & 9.4 & 39 & 3.50 \\
\hline & 0.96 & 8.9 & 35 & 3.00 \\
\hline & 1.00 & 8.8 & 38 & 3.32 \\
\hline & 0.98 & 9.0 & 38 & 3.34 \\
\hline & 0.98 & 9.5 & 39 & 3.61 \\
\hline & 0.99 & 9.1 & 39 & 3.51 \\
\hline & 0.99 & 9.4 & 38 & 3.54 \\
\hline & 0.98 & 9.2 & 38 & 3.42 \\
\hline \multirow{8}{*}{$3 \%(\mathrm{v} / \mathrm{v}) \mathrm{CN}$} & 0.99 & 10.7 & 41 & 4.32 \\
\hline & 0.99 & 10.6 & 43 & 4.52 \\
\hline & 0.99 & 10.3 & 42 & 4.33 \\
\hline & 0.98 & 10.6 & 40 & 4.15 \\
\hline & 0.99 & 10.4 & 42 & 4.36 \\
\hline & 0.99 & 10.2 & 42 & 4.21 \\
\hline & 1.00 & 8.8 & 45 & 3.93 \\
\hline & 0.99 & 10.2 & 42 & 4.26 \\
\hline \multirow[t]{5}{*}{$5 \%(\mathrm{v} / \mathrm{v}) \mathrm{CN}$} & 0.99 & 9.6 & 41 & 3.89 \\
\hline & 1.00 & 9.2 & 44 & 4.10 \\
\hline & 1.00 & 9.3 & 45 & 4.16 \\
\hline & 0.98 & 9.3 & 42 & 3.82 \\
\hline & 0.99 & 9.4 & 43 & 3.99 \\
\hline \multirow{6}{*}{$\begin{array}{l}\text { Thermal annealing, } \\
150^{\circ} \mathrm{C}, 10 \mathrm{~min}\end{array}$} & 0.94 & 4.0 & 37 & 1.40 \\
\hline & 0.93 & 3.6 & 38 & 1.28 \\
\hline & 0.93 & 3.7 & 38 & 1.31 \\
\hline & 0.92 & 3.9 & 38 & 1.36 \\
\hline & 0.91 & 3.5 & 38 & 1.22 \\
\hline & 0.93 & 3.8 & 38 & 1.31 \\
\hline \multirow{5}{*}{$\begin{array}{l}\text { Hot casting } \\
100^{\circ} \mathrm{C}\end{array}$} & 0.97 & 5.8 & 42 & 2.36 \\
\hline & 0.97 & 5.0 & 43 & 2.06 \\
\hline & 0.97 & 5.1 & 42 & 2.09 \\
\hline & 0.99 & 4.0 & 41 & 1.63 \\
\hline & 0.98 & 5.0 & 42 & 2.03 \\
\hline \multicolumn{5}{|l|}{$\begin{array}{l}o-\mathrm{DCB}, 15 \mathrm{mg} / \mathrm{mL} \text {, } \\
\text { 3:7 ratio }\end{array}$} \\
\hline \multirow[t]{7}{*}{ No additive } & 0.97 & 3.7 & 42 & 1.50 \\
\hline & 0.98 & 3.4 & 41 & 1.40 \\
\hline & 0.98 & 3.5 & 41 & 1.43 \\
\hline & 0.97 & 3.4 & 37 & 1.22 \\
\hline & 0.99 & 3.1 & 41 & 1.24 \\
\hline & 0.97 & 3.1 & 41 & 1.25 \\
\hline & 0.98 & 3.4 & 40 & 1.34 \\
\hline \multicolumn{5}{|l|}{$\begin{array}{l}o-\mathrm{DCB}, 15 \mathrm{mg} / \mathrm{mL}, \\
\text { 7:3 ratio }\end{array}$} \\
\hline \multirow[t]{7}{*}{ No additive } & 1.00 & 5.3 & 40 & 2.12 \\
\hline & 1.00 & 5.1 & 39 & 1.98 \\
\hline & 1.01 & 4.8 & 39 & 1.90 \\
\hline & 0.99 & 5.1 & 39 & 1.99 \\
\hline & 0.99 & 5.0 & 39 & 1.96 \\
\hline & 0.99 & 5.1 & 41 & 2.05 \\
\hline & 1.00 & 5.1 & 40 & 2.00 \\
\hline
\end{tabular}




\section{References}

(1) Pommerehne, J.; Vestweber, H.; Guss, W.; Mahrt, R. F.; Bässler, H.; Porsch, M.; Daub, J. Efficient Two Layer Leds on a Polymer Blend Basis. Adv. Mater. 1995, 7 (6), 551-554. https://doi.org/10.1002/adma.19950070608.

(2) Sun, Y.; Seo, J. H.; Takacs, C. J.; Seifter, J.; Heeger, A. J. Inverted Polymer Solar Cells Integrated with a Low-Temperature-Annealed Sol-Gel-Derived ZnO Film as an Electron Transport Layer. Adv. Mater. 2011, 23 (14), 1679-1683. https://doi.org/10.1002/adma.201004301.

(3) Frisch, M. J.; Trucks, G. W.; Schlegel, H. B.; Scuseria, G. E.; Robb, M. A.; Cheeseman, J. R.; Scalmani, G.; Barone, V.; Petersson, G. A.; Nakatsuji, H.; Li, X.; Caricato, M.; Marenich, A. V.; Bloino, J.; Janesko, B. G.; Gomperts, R.; Mennucci, B.; Hratchian, H. P.; Ortiz, J. V.; Izmaylov, A. F.; Sonnenberg, J. L.; Williams-Young, D.; Ding, F.; Lipparini, F.; Egidi, F.; Goings, J.; Peng, B.; Petrone, A.; Henderson, T.; Ranasinghe, D.; Zakrzewski, V. G.; Gao, J.; Rega, N.; Zheng, G.; Liang, W.; Hada, M.; Ehara, M.; Toyota, K.; Fukuda, R.; Hasegawa, J.; Ishida, M.; Nakajima, T.; Honda, Y.; Kitao, O.; Nakai, H.; Vreven, T.; Throssell, K.; Montgomery, J. A., Jr.; Peralta, J. E.; Ogliaro, F.; Bearpark, M. J.; Heyd, J. J.; Brothers, E. N.; Kudin, K. N.; Staroverov, V. N.; Keith, T. A.; Kobayashi, R.; Normand, J.; Raghavachari, K.; Rendell, A. P.; Burant, J. C.; Iyengar, S. S.; Tomasi, J.; Cossi, M.; Millam, J. M.; Klene, M.; Adamo, C.; Cammi, R.; Ochterski, J. W.; Martin, R. L.; Morokuma, K.; Farkas, O.; Foresman, J. B.; Fox, D. J Gaussian 09 Revis. E01 Gaussian Inc Wallingford CT.

(4) Wanwong, S.; Poe, A.; Balaji, G.; Thayumanavan, S. The Effect of Heteroatom Conformation on Optoelectronic Properties of Cyclopentadithiophene Derivatives. Org. Biomol. Chem. 2014, 12 (15), 2474-2478. https://doi.org/10.1039/C3OB41648H.

(5) Mora, J. U.; Garcia, I.; Zimmermann, I.; Aragó, J.; Calbo, J.; Grancini, G.; Ontoria, A. M.; Orti, E.; Martín, N.; Nazeeruddin, M. K. Saddle-like, $\pi$-Conjugated, Cyclooctatetrathiophene-Based, Hole-Transporting Material for Perovskite Solar Cells. J. Mater. Chem. C 2019. https://doi.org/10.1039/C9TC00437H.

(6) Wang, Y.; Wang, Z.; Zhao, D.; Wang, Z.; Cheng, Y.; Wang, H. Efficient Synthesis of Trimethylsilyl-Substituted Dithieno[2,3-b:3',2'-d]Thiophene, Tetra[2,3-Thienylene] and Hexa[2,3-Thienylene] from Substituted [3,3']Bithiophenyl. Synlett 2007, 2007 (15), 23902394. https://doi.org/10.1055/s-2007-985582.

(7) Hendsbee, A. D.; Sun, J.-P.; Law, W. K.; Yan, H.; Hill, I. G.; Spasyuk, D. M.; Welch, G. C. Synthesis, Self-Assembly, and Solar Cell Performance of N-Annulated Perylene Diimide Non-Fullerene Acceptors. Chem. Mater. 2016, 28 (19), 7098-7109. https://doi.org/10.1021/acs.chemmater.6b03292. 